\title{
1 Analysis of viral RNA-host protein interactomes 2 enables rapid antiviral drug discovery
}

3 Shaojun Zhang ${ }^{1,5}$, Wenze Huang ${ }^{1,5}$, Lili Ren ${ }^{2,3,5}$, Xiaohui Ju ${ }^{4,5}$, Mingli Gong,

4 Jian Rao ${ }^{2,3,5}$, Lei Sun ${ }^{1}$, Pan Li ${ }^{1}$, Qiang Ding ${ }^{4, *}$, Jianwei Wang ${ }^{2,3, *}$, Qiangfeng Cliff 5 Zhang ${ }^{1, *}$

$7 \quad{ }^{1}$ MOE Key Laboratory of Bioinformatics, Beijing Advanced Innovation Center for 8 Structural Biology \& Frontier Research Center for Biological Structure, Center for 9 Synthetic and Systems Biology, Tsinghua-Peking Joint Center for Life Sciences,

10 School of Life Sciences, Tsinghua University, Beijing, 100084, China

$11{ }^{2}$ NHC Key Laboratory of Systems Biology of Pathogens and Christophe Mérieux

12 Laboratory, Institute of Pathogen Biology, Chinese Academy of Medical Sciences \&

13 Peking Union Medical College, Beijing, 100730, China

$14{ }^{3}$ Key Laboratory of Respiratory Disease Pathogenomics, Chinese Academy of Medical

15 Sciences and Peking Union Medical College, Beijing, 100730, China

$16{ }^{4}$ Center for Infectious Disease Research, School of Medicine, Tsinghua University,

17 Beijing, 100084, China

$18{ }^{5}$ Co-first author

19 * Correspondence: qding@ tsinghua.edu.cn (Q.D.), wangjw28@ 163.com (J.W.),

20 qczhang@tsinghua.edu.cn (Q.C.Z.)

21 Short title: Viral RNA and host protein interactome

22 Keywords: RNA-protein interaction, SARS-CoV-2, EBOV, ZIKV, drug discovery 


\section{Abstract}

25 RNA viruses including SARS-CoV-2, Ebola virus (EBOV), and Zika virus (ZIKV)

26 constitute a major threat to global public health and society. The interactions

27 between viral genomes and host proteins are essential in the life cycle of RNA

28 viruses and thus provide targets for drug development. However, viral RNA-host

29 protein interactions have remained poorly characterized. Here we applied

30 ChIRP-MS to profile the interactomes of human proteins and the RNA genomes

31 of SARS-CoV-2, EBOV, and ZIKV in infected cells. Integrated interactome

32 analyses revealed interaction patterns that reflect both common and

33 virus-specific host responses, and enabled rapid drug screening to target the

34 vulnerable host factors. We identified Enasidenib as a SARS-CoV-2 specific

35 antiviral agent, and Trifluoperazine and Cepharanthine as broad spectrum

36 antivirals against all three RNA viruses.

38 One Sentence Summary:

39 Interactome analyses of host proteins and the SARS-CoV-2, EBOV, and ZIKV RNA

40 genomes unveil viral biology and drug targets. 


\section{Introduction}

It is notable that three of the most dangerous infectious diseases of recent times are all caused by RNA viruses: SARS-CoV-2, ZIKV and EBOV. The SARS-CoV-2 coronavirus has caused the on-going Coronavirus Disease 2019 (COVID-19) pandemic, resulting in more than 20 million infections and 800 thousand deaths and global disruption of our society and economy $(1,2)$. Since its outbreak in 2012, ZIKV has infected millions of people(3), and has remained on the 2018 Blueprint list of priority diseases by the World Health Organization (WHO, February, 2018). EBOV has led to epidemics in Africa and has spread to Europe and North America; Ebola virus disease is severe with an average case fatality rate of $\sim 80 \%$ in past outbreaks(4).

RNA viruses typically have a relatively small genome and proteome(5), and therefore rely heavily on interactions with host factors to complete their life cycles $(\sigma)$. RNA binding proteins function on many aspects of cellular and viral processing, e.g., RNA translation, stabilization, modification and $\operatorname{localization}(7,8)$, as well as anti-infection response against pathogens(9). Many studies have focused characterization of viral protein-host protein interactions(10-15). In contrast, the interactions between host proteins and viral RNA (vRNA) are much less well understood, despite the known importance of the viral RNA genome for multiple processes during infection, including viral genome translation and replication(16).

Recent years have seen an explosion of high-throughput methods that, in combination with diverse crosslinking reagents and data processing workflows, enable a global analysis of RNA-protein interactions ("the interactome") in cells $(17,18)$. These approaches can substantially advance our understanding of the infection and pathology of RNA viruses and can inform diverse and effective therapeutic options, yet they have not yet been widely deployed for comparative analyses of cells infected with diverse RNA viruses or strains.

Here, we unveil the vRNA-host protein interactomes of SARS-CoV-2, EBOV, 
and ZIKV based on ChIRP-MS analysis of infected human host cells. Our integrated interactome analysis identified interaction patterns that reflect both common and virus-specific host responses. We applied these insights to inform a targeted antiviral drug screening workflow based on standard and/or trans-complementation viral infection assay systems that ultimately identified the FDA approved drug Enasidenib, which inhibits the SARS-CoV-2 specific interacting protein IDH2, as a potent SARS-CoV-2 specific antiviral agent. Further, we found that the heat shock protein inhibitor Cepharanthine and the cytoskeleton disruptor Trifluoperazine are broad spectrum antivirals against all three of these pathogenic RNA viruses.

\section{Results}

\section{ChIRP-MS reveals the SARS-CoV-2 vRNA-host protein interactome}

To define host proteins associated with genomic RNA of SARS-CoV-2, we used the RNA-directed proteomic discovery method ChIRP-MS (Comprehensive identification of RNA-binding proteins by mass spectrometry)(19) (Materials and Methods). Briefly, human hepatocarcinoma Huh7.5.1 cells were infected with SARS-CoV-2 and crosslinked with formaldehyde to preserve vRNA-protein complexes. Biotinylated oligonucleotides tiling SARS-CoV-2 vRNA with specificity were used to enrich vRNA-host protein complexes from cell lysates (Table S1) and the co-purified proteins were identified by mass spectrometry (Fig. 1A).

ChIRP-MS analysis of SARS-CoV-2-infected Huh7.5.1 cells identified a total of 143 human proteins with a MiST score > 0.6 (Fig. 1B and Table S2). Note that the co-purification steps of the ChIRP-MS strategy depleted highly abundant host RNAs and enabled robust recovery of over $80 \%$ of the total viral RNA in infected cells (Fig. S1A). Correlation coefficients across three biological replicates were all above 0.9 (Fig. 1C). Our ChIRP-MS dataset underscored the extensive interactions between viral RNA and the SARS-CoV-2 nucleocapsid protein (NP), the viral M protein, the spike protein 
and several non-structural proteins (NS) (Table S2. Also see Fig. 1B), consistent with previous studies showing that NP directly binds and encases viral RNA during infection.

To validate the vRNA-interacting host proteins from the mass spectrometry based identification, we assessed the ChIRP samples using western blotting (ChIRP-WB) (Fig. 1A. Materials and Methods). We examined 11 candidate proteins using antibodies that were available in our lab (e.g., HnRNPU and PPIA with a high MiST score 1, and RACK1 with a lower MiST score 0.68), and confirmed that 9 of them as SARS-CoV-2 vRNA interacting proteins in infected Huh7.5.1 cells (Fig. 1D). Among them, ELVAL1, hnRNPU, and PTBP1 were previously revealed to bind other coronavirus vRNAs that function in viral replication $(20,21)$. PPIA (Cyclophilin A) has been widely implicated in viral infection, and drugs targeting PPIA showed broad-spectrum antiviral activity(22). MATR3 may associate with SFPQ/NONO heterodimers to modulate the STING pathway(23). IGF2BP1 is a well-characterized RNA binding protein involved in RNA stability and viral RNA translation(24). The THO complex subunit protein (ALYREF) is a component of the TREX complex that is involved in RNA processing and transport(25). Offering further support that our approach yielded biologically relevant candidates rather than processing-related artefacts, we noted that the vRNA-interacting candidate host proteins were enriched for canonical RNA-binding domains (e.g., RNA recognition motif, RNA secondary structure related DEAD/DEAH box helicase domain, and RNA-binding KH domain) (Fig. 1E, Fig. S1B).

We comapared our 143 SARS-CoV-2 vRNA binding proteins with host factors previously reported to bind coronaviruses, and found that 14 of the host factors in our ChIRP-MS interactome are known to bind the viral RNA of other coronavirus and to impact viral replication(20, 21) (Fig. 1B). In particular, we comapared our dataset with a recently reported host interactome for SARS-CoV-2 based on the "RNA antisense purification and mass spectrometry" (RAP-MS) method(26), and found 17 host factors 
124 common to both interactome datasets (Fig. S1C). In addition, of the 143 SARS-CoV-2

125 host RNA binding proteins that we identified, 69 (61\%, Table S2) were previously

126 reported as responsive to SARS-CoV-2 infection in a proteomics study(27).

127 Collectively, these findings suggest that ChIRP-MS can reliably identify

128 vRNA-interacting host factors to thus to catalog a high-confidence SARS-CoV-2

129 vRNA interactome.

130 SARS-CoV-2 infects multiple organs, leading to diverse symptoms such as fever,

131 shortness of breath, severe acute pneumonia, haempotysis, diarrhea, and neurological

132 disorders(28). Indeed, we found that many of the SARS-CoV-2 vRNA-binding

133 proteins were more highly expressed in organs known to host SARS-CoV-2 infection,

134 such as lung(29), intestines(30) and brain(31) (Fig. 1F-G). We also performed Gene

135 Ontology (GO) and Kyoto Encyclopedia of Genes and Genomes (KEGG) analyses and

136 found that the annotations of the interactome host factors were enriched for GO terms

137 related to splicing, translation, and viral transcription (Fig. 1H), as well as KEGG

138 pathways related to the spliceosome, ribosome and RNA metabolism (Fig. 1I). The

139 aforementioned proteomics survey also reported that host proteins responsive to

140 SARS-CoV-2 infection are similarly enriched for annotated functions relating to

141 translation, RNA metabolism, and RNA transport related cellular processes(27).

142

\section{High-confidence vRNA-human protein interactomes for EBOV and ZIKV}

144 Seeking to delineate which host proteins are common to different RNA viruses vs.

145 virus-specific factors, we used ChIRP-MS to uncover the vRNA-host protein 146 interactomes for the ZIKV MR766 strain and a recombinant EBOVDVP30-GFP virus

147 with the VP30 coding sequence of the Ebola virus (strain Zaire Mayinga) replaced by

148 the GFP sequence(32) (Materials and Methods). As above, we employed biotinylated

149 oligonucleotides tiling each viral RNA with specificity to enrich for viral RNA-host

150 protein complexes from lysates of infected cells (Table S1), and identified the

151 co-purified proteins by mass spectrometry (Fig. 1A). 
These ChIRP-MS analyses identified a total 172 viral RNA binding proteins in

153 MR766-infected Huh7 cells (MiST score > 0.7) and 223 viral RNA binding proteins in

154 EBOVDVP30-GFP infected Huh7.5.1-VP30 cells (MiST score > 0.6, Fig. 2A-B and

155 Table S2). In each analysis, the co-purification steps of the ChIRP-MS strategy allowed

156 for the depletion of highly abundant host RNAs, and, as with SARS-CoV-2, enabled

157 robust recovery of more than $80 \%$ of the total viral RNA in infected cells (Fig. S2A).

158 All data showed high correlations between the experimental replicates $(r>0.7$ and 0.9

159 respectively for MR766 and EBOV, Fig. 2B-C).

160 We again used ChIRP-WB to verify six of the host RNA binding proteins 161 uncovered by ChIRP-MS for both ZIKV and EBOV (IGF2BP1, ALYREF, SFPQ, 162 MATR3, PDIA6 and SND1) and validated their interactions with viral RNAs in 163 ZIKV-infected Huh7 cells and in EBOV infected Huh7.5.1 cells (Fig. 2C). With only 164 one exception (interaction between SND1 and EBOV vRNA), all validation results 165 were consistent with our ChIRP-MS results. We then examined 12 host proteins that 166 were shown previously to interact with various ZIKV strains, 8 of these 12 host 167 proteins passed our scoring cutoff, suggesting a very high sensitivity for our 168 ChIRP-MS experiments (Table S2). In addition, comparisons against previously

169 reported ChIRP-MS based studies of host proteins that interact with another ZIKV 170 strain(17) also revealed high level of overlap (Fig. S2D). Similarly, we also examined 171 proteins that previously reported to interact with EBOV vRNA (DHX9, hnRNPR, 172 hnRNPL, SYNCRIP, IGF2BP1)(33), and confirmed that all these interactions are 173 covered in our EBOV ChIRP-MS result (Table S2). Overall, these analysis and 174 observations suggest that our ChIRP-MS reliably captured host proteins that interact 175 with the ZIKV and EBOV vRNAs in infected cells. 


\section{An integrated analysis of the three RNA virus host factor interactomes identifies}

178 common and virus-specific host cellular factors and responses

179 Our interactome datasets highlight that the three RNA viruses interact with many 180 common host proteins, but also showcase their virus-specific host interaction partners.

181 In total, 33 human proteins interact with viral RNAs of all three viruses, and 88 proteins

182 interact with viral RNAs of two of the three viruses, results suggesting the potential 183 involvement of common host factors in conserved host reponses to infection by RNA 184 viruses (Fig. 2D). Note that compared to the total set of all vRNA-interacting proteins, 185 these common interacting proteins exhibit stronger enrichment for canonical 186 RNA-binding domains (Fig. 2E), and consistently showed enrichment of cellular 187 functions such as splicing, translation, and metabolism related processings (Fig. 2F).

188 To further characterize conserved host responses to RNA virus infection, we 189 examined the common interacting protein complexes (and protein associations of the 190 same biological pathways) shared by the three RNA viruses. We searched for enriched 191 protein complexes and defined a complex as common one if it was present in at least 192 two of the three virus interactomes (Fig. 3. Table S3. Materials and Methods). The 193 analysis identified many common protein complexes, including the ribosome (and 194 related translation regulators such as eIF4A), the spliceosome (and also other RNA 195 processing proteins such as YBX1 and RBMX), the IGF2BP1-associated complex, the 196 large drosha and DGCR8 complexes, the TNF-alpha/NF-kB signaling complex, the 197 microtubules and the cytoskeleton, the proteasome, and many stress-granule-related 198 proteins.

199 Many of these common complexes are known to function in the regulation of host 200 cell and/or viral RNA. For example, the IGF2BP1-associated complex regulates the 201 stability and translation of various cellular RNA molecules including c-Myc(34) and 202 also enhances IRES-mediated translation initiation of HCV RNA(24). The large drosha 203 and DGCR8 complexes, which are involved in miRNA biogenesis, are essential for 204 degrading exogenous double-stranded RNA, including viral RNAs(35). The 
205 cytoskeleton is often rearranged by viral infection and is involved in the formation of

206 virus-induced membranous replication factories and that chemicals targeting the 207 cytoskeleton are known to inhibit the replication of RNA virus(36). Of particular 208 interests, host factors that function as stress-granule-related proteins(37), were 209 repeatedly observed in the interactomes of all three viruses (Fig. 3), supporting the idea 210 that stress granules as a general host cell response to viral infection.

211 Of equally paramount interest, we also identified many virus-specific protein 212 complexes and pathways (Fig. 3, Fig. 3A-F, Table S3). For example, the SARS-CoV-2 213 vRNA interactome was specifically enriched for two metabolism pathways: the 214 tricarboxylic acid (TCA)-cycle and the 2-Oxocarboxylic acid metabolism (i.e., lipid 215 metabolsim). Indeed, previous proteomic investigation also observed that these 216 pathways are disrupted by SARS-CoV-2 infection(27). We also discovered that ZIKV 217 interacts with components of the TREX transcription/export complex (comprising 218 THOCs, ALYREF, DDX39B and SARNP, Fig. 3). This protein complex is important 219 for RNA transport and its disruption has been implicated in microcephaly(38, 39), a 220 disease associated with ZIKV infection(40). The SARS-CoV-2 host factor interactome 221 includes only two known THOC complex subunits, while the EBOV includes none. We 222 also found that EBOV specifically interacts with a set of immune and inflammatory 223 responses related proteins(41-43), including S100A9, ANXA1, and DDX3X, and 224 especially the "IFN- $\gamma$-activated inhibitor of translation" (GAIT) complex (Fig. 3), 225 which is known to repress the translation of inflammatory related mRNAs(44). At 226 minimum, these findings warrant further investigation for a functional connection to 227 the organ failure and death that arise from the dysregulated host inflammatory 228 responses during EBOV infection(45). 


\section{Interactome-informed drug repurposing discovered potent SARS-CoV-2}

\section{1 antiviral compounds}

232 The selective inhibition of host vRNA-binding proteins can attenuate infection and

233 inform the development of innovative therapies(12). Accordingly, existing drugs that

234 are known to target various host factor proteins can be exploited as potential treatments

235 strategies during epidemics and pandemics. Given the urgent need for COVID-19

236 therapies(46), we analyzed open-source chemical databases to uncover compounds that

237 target host factors within the SARS-CoV-2 vRNA interactome. Specifically, we

238 investigated the IUPHAR/BPS Guide to Pharmacology, Drugbank, Drugcentral, and

239 ChEMBL databases, and identified a total of 5309 compounds (Table S4) that are

240 known or predicted to interact with 56 SARS-CoV-2 vRNA-binding proteins. We

241 prioritized approved drugs and clinical phase agents, as well as those available at the

242 Center of Pharmaceutical Technology of Tsinghua University. Ultimately, we tested

243 the antiviral activities of 21 drugs (Fig. 4A, Table S5), including 20 FDA-approved

244 drugs and 1 agent (Silvestrol) being tested in preclinical trials.

245 To efficiently assess the potential antiviral effects on SARS-CoV-2 infection for 246 these drugs, we used a trans-complementation system based on human colorectal 247 adenocarcinoma epithelial Caco2 cells that supports infection and replication of 248 SARS-CoV-2 (Fig. 4B). Briefly, this system uses a SARS-CoV-2-GFP $\Delta$ N genome 249 wherein the N protein-coding sequence is replaced with GFP; this replacement both 250 disrupts the packaging capacity of the virus and supports visualization. 251 SARS-CoV-2-GFP $\Delta \mathrm{N}$ vRNA was generated by in vitro transcription and transfected 252 into green monkey epithelial Vero cells that stably express the SARS-CoV-2 N protein. 253 These steps yielded packaged SARS-CoV-2-GFP $\Delta \mathrm{N}$ virions, which we used to infect 254 Caco2 cells that also stably express the SARS-CoV-2 N protein (Caco2-N). We used 255 GFP expression as a surrogate to monitor viral infection and replication in human cells. 256 We treated Caco2-N cells with each drug at the same time as the viral challenge. 257 Remdesivir, which targets the viral RdRp and is well known for its antiviral activity 
258 against SARS-CoV-2, was used as a positive control, and vehicle (DMSO) was used as

259 a negative control. At $72 \mathrm{~h}$ post infection we quantified infected, GFP-positive cells by

260 FACS. Remdesivir $(2 \mu \mathrm{M})$ was found to strongly inhibit SARS-CoV-2-GFP $\Delta \mathrm{N}$

261 infection, with an infection about $3.7 \%$ relative to the negative control DMSO-treated

262 cells (Fig. 4C). Thus, our trans-complementation system reflects SARS-CoV-2

263 replication in cells and is suitable for initial drug-testing.

264 Our initial screen using $10 \mu \mathrm{M}$ of each selected compound uncovered activity 265 against SARS-CoV-2 for 15 of the 21 tested candidate drugs (Fig. 4C). Five exerted 266 strong antiviral effects (Fig. 4C): 1) Silvestrol, which targets the translation initiation 267 factor eIF4A(47) (preclinical on Chronic lymphocytic leukemia; 98.8\% inhibitory 268 efficiency); 2), Cyclosporin A (CsA), which is an immunosuppression drug and targets 269 protein PPIA(48) (FDA-approved for immunosuppressant; 91.2\% inhibitory 270 efficiency); 3), Trifluoperazine (FDA-approved for antipsychotic and an antiemetic; $27187.5 \%$ inhibitory efficiency) disrupts cytoskeleton organization(49) and also inhibits 272 binding between HSP90 with its chaperons(50), and 4) Cepharanthine (Approved for 273 alopecia, $89.6 \%$ inhibitory efficiency), which is an inhibitor of TNF-alpha/NF-kB, and 274 also an inhibitor of the heat shock proteins (HSP90s and HSPA8)(51, 52) and 5) 275 Enasidenib, which targets the mitochondrial energy related enzyme IDH2(53) 276 (FDA-approved for refractory acute myeloid leukemia; 58\% inhibitory efficiency).

277 Despite the potential for inhibition of SARS-CoV-2 infection at the initial $10 \mu \mathrm{M}$ 278 concentrations, these five candidate drugs also showed varying levels of cytotoxicity 279 (Fig. S4A). We further used the trans-complementation assays to determine the 280 antiviral potency and cytotoxicity at different concentrations for the aforementioned 281 five candidates (Fig. 4D). For testing the drug effects, we infected Caco-2 cells with 282 SARS-CoV-2 virus (MOI 0.05) and treated cells with the compounds with different 283 concentrations. Remdesivir, as positive control, reduced the infection of SARS-CoV-2 284 virus with an IC50 value of $0.03 \mu \mathrm{M}$ (Fig. S4B). Silvestrol was previously reported as a 285 "broad-spectrum" antiviral agent that showed inhibition activity against the 
286 coronavirus HCoV-229E(54). Although Silvestrol showed a dose-dependent effect on

287 SARS-CoV-2 infection (IC50=0.32 $\mu \mathrm{M}$ ), we found that the cell viabilities dropped 288 dramatically at higher drug concentrations, and detected no inhibitory effects on 289 infection of SARS-CoV-2 virus infection below a 100nM of Silvestrol concentration.

290 CsA can inhibit infection of SARS-CoV-2 virus with an IC50 value of $3.8 \mu \mathrm{M}$, and a

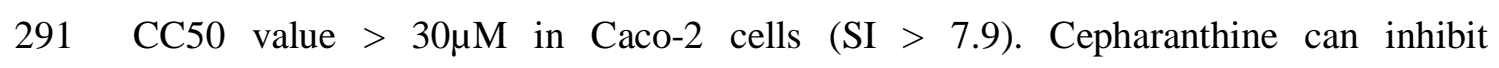
292 SARS-CoV-2 infection with an IC50 value of $2.98 \mu \mathrm{M}$, and a CC50 value of $31.77 \mu \mathrm{M}$ 293 (SI=10.66). Trifluoperazine also showed strong antiviral activities against 294 SARS-CoV-2, with IC50 value of $3.15 \mu \mathrm{M}$, and CC50 value > 30 $\mu \mathrm{M}$ ( $\mathrm{SI}>9.51)$. 295 Enasidenib, targeting the SARS-CoV-2 vRNA specific host factor IDH2, showed no 296 antiviral effects. Thus, among the tested candidate drugs, Cepharanthine (approved in 297 Japan) and Trifluoperazine (FDA-approved) are the most attractive for potential use for 298 against SARS-CoV-2 infection.

300 Identification of broad antiviral inhibitors against multiple RNA viruses by 301 targeting common host factors

302 Our viral RNA interactome data indicated that both ZIKV and EBOV vRNAs interact 303 with the translational machinery, heat shock proteins (e.g., HSP90s, HSPA9, HSPA8 304 HSPD1, etc.), as well as cytoskeleton-related proteins (e.g., TUBA1C, TUBB). This 305 overlap amongst host factors for these RNA viruses motivated us to examine the 306 antiviral effects of drugs targeting these host proteins, specifically using infection 307 assays for ZIKV in Huh7 cells (MR766, MOI 0.5) and for EBOV using assays in in 308 Huh7.5.1-VP30 cells based on an EBOVAVP30-GFP variant(32) (Fig. 4E-F).

309 The translation initiation inhibitor Silvestrol inhibited ZIKV infection with an 310 IC50 value of $0.01 \mu \mathrm{M}$ and a CC50 value of $0.04 \mu \mathrm{M}$ (SI=4). CsA inhibited ZIKV 311 infection with an IC50 value of $0.78 \mu \mathrm{M}$, and a CC50 value of $14.35 \mu \mathrm{M}(\mathrm{SI}=18.40)$. 312 And the cytoskeleton-disrupter Trifluoperazine inhibited ZIKV infection with an IC50 313 value of $0.96 \mu \mathrm{M}$, and a CC50 value of $7.79 \mu \mathrm{M}$ (SI=8.11). The most potent anti-ZIKV 
314 effects we observed in these assays was from Cepharanthine that targets heat shock

315 proteins, which inhibited ZIKV infection with an IC50 value of $2.19 \mu \mathrm{M}$, and a CC50

316 value of $24 \mu \mathrm{M}(\mathrm{SI}=10.97)$ (Fig. 4E). And Enasidenib targeting IDH2 showed low

317 inhibitory effects on ZIKV infection $(\mathrm{SI}=1.84)$.

318 As a negative-strand RNA virus, the infection and replication processes of EBOV 319 are very distinct from those of positive-strand RNA virus such as SARS-CoV-2 and 320 ZIKV(4). Consistently, our integrated viral RNA interactome analysis for the three 321 RNA viruses indicated that SARS-CoV-2 and ZIKV share more overlapped host 322 factors than that of any with EBOV, specifically, PPIA interacts with vRNAs of 323 SARS-CoV-2 and ZIKV, while not with vRNA of EBOV. Indeed, the PPIA-inhibitor 324 CsA showed antiviral activities against SARS-CoV-2 and ZIKV but not 325 EBOV $\Delta$ VP30-GFP viral infection (MOI 0.1), even at a high concentration (10 $\mu \mathrm{M}$, Fig. 326 4D-F). This result is also consistent with a previous study reporting that another PPIA 327 inhibitor (Alisporivir) has limited antiviral activity against EBOV(55). Again, 328 Silvestrol showed obvious cell toxicity although it exerted dose-dependent inhibition to 329 EBOV $\Delta$ VP30-GFP infection (IC50 $=0.02 \mu \mathrm{M}$ ), and Trifluoperazine (IC50 = $1.79 \mu \mathrm{M}$, $330 \mathrm{SI}=2.18)$ and Cepharanthine $(\mathrm{IC} 50=0.42 \mu \mathrm{M}, \mathrm{SI}=12.10)$ showed antiviral activities 331 to EBOVAVP30-GFP virus infection. The IDH2 inhibitor Enasidenib also showed no 332 effects on EBOV $\triangle$ VP30-GFP virus infection. Note that in our study here, we used 333 SARS-CoV-2 for initial test of the antivirals, which could potentially miss EBOV 334 specific antivirals, given the distinct life cycle of positive-strand and negative-strand 335 RNA viruses. It is worthy in the future to perform an EBOV host factor guided-large 336 scale screening for discovery of more effective antivirals against EBOV.

337 Collectively, our results support that the FDA-approved cytoskeleton disrupter 338 drug Trifluoperazine and the heat shock proteins inhibitor Cepharanthine have 339 broad-spectrum antiviral activity against the three major RNA viruses. 


\section{Conclusions}

342 Our ChIRP-MS profiling of the vRNA-host protein interactomes of human cells

343 infected with the causal pathogenic viruses for COVID-19, Zika and Ebola virus

344 diseases identified interaction patterns that reflect both common and virus-specific

345 host responses. These interactome datasets can be viewed as a rich resource to help

346 illuminate the infection biology for these three viral pathogens and potentially others as

347 well. The RNA-centric view of ChIRP-MS was particularly informative in revealing

348 molecular virology of all stages of the life cycle of RNA viruses in infected cells(17).

349 Methods to analyze RNA-protein interactome datasets are not yet standardized, and we

350 found that integrated comparisons offered a straightforward way to explore and

351 visualize common and virus-specific interactions, which we arranged to highlight the

352 functions of the various host proteins. Indeed, the analysis enabled the prediction and

353 selection of small molecule inhibitors as either specific or likely broad-spectrum

354 antiviral agents. And finally, we were able to combine the interactome-informed

355 predictions and an innovative trans-complementation infection system to power a

356 pipeline for rapid antiviral drug discovery.

357 Ultimately, these efforts demonstrated that the FDA-approved IDH2 inhibitor 358 Enasidenib is a potent SARS-CoV-2 specific antiviral agent and that agents targeting

359 the heat shock proteins and cytoskeleton components, Cepharanthine and

360 Trifluoperazine, can exert broad-spectrum effects against all three RNA viruses we

361 examined in our study. Having identified the activities of Enasidenib, Cepharanthine,

362 and Trifluoperazine, next steps for their re-purposing and deployment as possible

363 clinical antiviral agents would be in vivo infection model studies and pre-clinical

364 safety evaluations. Moreover, deeper exploration of the antiviral modes of action for

365 these drugs should reveal biological insights about how these viruses infect cells, how

366 host cells respond, and which (if any) countermeasures the viruses may deploy to

367 overcome host defense pathways. The strong current focus of the biomedical research

368 community on the biology of RNA virus infection, especially the ongoing COVID-19 
369 pandemic(56), has exposed how little we know about the infection and amplification

370 mechanisms that these viruses employ. Our work illustrates how profiling datasets

371 obtained with ChIRP-MS can be integrated to drive both basic discovery and

372 therapeutic applications.

373

\section{Reference and Notes}

375 1. L. Sainati, A. Biffi, How we deal with the COVID-19 epidemic in an Italian

376 paediatric onco-haematology clinic located in a region with a high density of

377 cases. Br. J. Haematol. 189, 640-642 (2020).

378 2. WHO, Coronavirus disease (COVID-2019) situation reports

379 https://www.who.int/emergencies/diseases/novel-coronavirus-2019/situation-r

380 eports/. (2020).

381 3. C. Shan et al., Zika Virus Vaccine: Progress and Challenges. Cell Host Microbe

$382 \quad$ 24, 12-17 (2018).

383 4. D. Malvy et al., Ebola virus disease. Lancet 393, 936-948 (2019).

384 5. D. Kim et al., The Architecture of SARS-CoV-2 Transcriptome. Cell 181, 385 914-921 e910 (2020).

386 6. P. D. Nagy, J. Pogany, The dependence of viral RNA replication on co-opted 387 host factors. Nat. Rev. Microbiol. 10, 137-149 (2011).

388 7. E. L. Van Nostrand et al., A large-scale binding and functional map of human 389 RNA-binding proteins. Nature 583, 711-719 (2020).

390 8. S. R. Gonzales-van Horn, P. Sarnow, Making the Mark: The Role of Adenosine 391 Modifications in the Life Cycle of RNA Viruses. Cell Host Microbe 21, 392 661-669 (2017).

393 9. X. Ren et al., RIG-I Recognition of RNA Targets: The Influence of Terminal 394 Base Pair Sequence and Overhangs on Affinity and Signaling. Cell Rep 29, $395 \quad 3807-3815$ e3803 (2019).

396 10. P. S. Shah et al., Comparative Flavivirus-Host Protein Interaction Mapping 397 Reveals Mechanisms of Dengue and Zika Virus Pathogenesis. Cell 175, 398 1931-1945 e1918 (2018).

399 11. J. Batra et al., Protein Interaction Mapping Identifies RBBP6 as a Negative $400 \quad$ Regulator of Ebola Virus Replication. Cell 175, 1917-1930.e1913 (2018).

401 12. D. E. Gordon et al., A SARS-CoV-2 protein interaction map reveals targets for 402 drug repurposing. Nature 583, 459-468 (2020).

403 13. K. Schubert et al., SARS-CoV-2 Nsp1 binds ribosomal mRNA channel to 404 inhibit translation. bioRxiv, 2020.2007.2007.191676 (2020).

406

14. M. Thoms et al., Structural basis for translational shutdown and immune 408

15. G. Lasso et al., A Structure-Informed Atlas of Human-Virus Interactions. Cell 178, 1526-1541 e1516 (2019). 
409

410

411

412

413

414

415

416

417

418

419

420

421

422

423

424

425

426

427

428

429

430

431

432

433

434

435

436

437

438

439

440

441

442

443

444

445

446

447

448

449

450

16. A. M. Boguszewska-Chachulska, A.-L. Haenni, RNA viruses redirect host factors to better amplify their genome. Adv. Virus Res. 65, 29-61 (2005).

17. Y. S. Ooi et al., An RNA-centric dissection of host complexes controlling flavivirus infection. Nat Microbiol 4, 2369-2382 (2019).

18. M. Ramanathan et al., RNA-protein interaction detection in living cells. Nat. Methods 15, 207-212 (2018).

19. C. Chu et al., Systematic Discovery of Xist RNA Binding Proteins. Cell 161, 404-416 (2015).

20. S. T. Shi, M. M. Lai, Viral and cellular proteins involved in coronavirus replication. Curr. Top. Microbiol. Immunol. 287, 95-131 (2005).

21. C. Galán et al., Host cell proteins interacting with the $3^{\prime}$ end of TGEV coronavirus genome influence virus replication. Virology 391, 304-314 (2009).

22. D. Zhou et al., Cyclophilin A and viral infections. Biochem. Biophys. Res. Commun. 424, 647-650 (2012).

23. M. Salton et al., Involvement of Matrin 3 and SFPQ/NONO in the DNA damage response. Cell Cycle 9, 1568-1576 (2010).

24. S. Weinlich et al., IGF2BP1 enhances HCV IRES-mediated translation initiation via the 3'UTR. RNA 15, 1528-1542 (2009).

25. C. G. Heath et al., The role of TREX in gene expression and disease. Biochem. J. 473, 2911-2935 (2016).

26. N. Schmidt et al., A direct RNA-protein interaction atlas of the SARS-CoV-2 RNA in infected human cells. bioRxiv, (2020).

27. D. Bojkova et al., Proteomics of SARS-CoV-2-infected host cells reveals therapy targets. Nature, (2020).

28. J. Helms et al., Neurologic Features in Severe SARS-CoV-2 Infection. New Engl. J. Med. 382, 2268-2270 (2020).

29. N. Zhu et al., A Novel Coronavirus from Patients with Pneumonia in China, 2019. N Engl J Med 382, 727-733 (2020).

30. M. M. Lamers et al., SARS-CoV-2 productively infects human gut enterocytes. Science 369, 50 (2020).

31. B. Z. Zhang et al., SARS-CoV-2 infects human neural progenitor cells and brain organoids. Cell Res., (2020).

32. P. Halfmann et al., Generation of biologically contained Ebola viruses. Proc Natl Acad Sci U S A 105, 1129-1133 (2008).

33. J. Fang et al., Staufen1 Interacts with Multiple Components of the Ebola Virus Ribonucleoprotein and Enhances Viral RNA Synthesis. mBio 9, e 01771-01718 (2018).

34. D. Weidensdorfer et al., Control of c-myc mRNA stability by IGF2BP1-associated cytoplasmic RNPs. RNA 15, 104-115 (2009).

35. S. K. Pong, M. Gullerova, Noncanonical functions of microRNA pathway enzymes - Drosha, DGCR8, Dicer and Ago proteins. FEBS Lett. 592, 2973-2986 (2018). 
451

452

453

454

455

456

457

458

459

460

461

462

463

464

465

466

467

468

469

470

471

472

473

474

475

476

477

478

479

480

481

482

483

484

485

486

487

488

489

490

491

36. M. Cortese et al., Ultrastructural Characterization of Zika Virus Replication Factories. Cell Rep. 18, 2113-2123 (2017).

37. S. Markmiller et al., Context-Dependent and Disease-Specific Diversity in Protein Interactions within Stress Granules. Cell 172, 590-604.e513 (2018).

38. R. Kumar et al., THOC2 Mutations Implicate mRNA-Export Pathway in X-Linked Intellectual Disability. Am. J. Hum. Genet. 97, 302-310 (2015).

39. C. L. Beaulieu et al., Intellectual disability associated with a homozygous missense mutation in THOC6. Orphanet J Rare Dis 8, 62 (2013).

40. J. Mlakar et al., Zika Virus Associated with Microcephaly. New Engl. J. Med. 374, 951-958 (2016).

41. S. Yui et al., Calprotectin (S100A8/S100A9), an inflammatory protein complex from neutrophils with a broad apoptosis-inducing activity. Biol. Pharm. Bull. 26, 753-760 (2003).

42. V. Ferlazzo et al., Anti-inflammatory effects of annexin-1: stimulation of IL-10 release and inhibition of nitric oxide synthesis. Int. Immunopharmacol. 3, 1363-1369 (2003).

43. H. Oshiumi et al., DEAD/H BOX 3 (DDX3) helicase binds the RIG-I adaptor IPS-1 to up-regulate IFN-beta-inducing potential. Eur. J. Immunol. 40, 940-948 (2010).

44. R. Mukhopadhyay et al., The GAIT system: a gatekeeper of inflammatory gene expression. Trends Biochem. Sci. 34, 324-331 (2009).

45. L. Baseler et al., The Pathogenesis of Ebola Virus Disease. Annu Rev Pathol 12, 387-418 (2017).

46. R. K. Guy et al., Rapid repurposing of drugs for COVID-19. Science 368, 829-830 (2020).

47. C. Müller et al., Broad-spectrum antiviral activity of the eIF4A inhibitor silvestrol against corona- and picornaviruses. Antiviral Res. 150, 123-129 (2018).

48. F. Yang et al., Cyclophilin A Is an Essential Cofactor for Hepatitis C Virus Infection and the Principal Mediator of Cyclosporine Resistance In Vitro. $J$. Virol. 82, 5269-5278 (2008).

49. Q. Y. Chen et al., Molecular mechanism of trifluoperazine induces apoptosis in human A549 lung adenocarcinoma cell lines. Mol Med Rep 2, 811-817 (2009).

50. Y. M. Ning, E. R. Sanchez, Evidence for a Functional Interaction Between Calmodulin and the Glucocorticoid Receptor. Biochem. Biophys. Res. Commun. 208, 48-54 (1995).

51. Y. B. Zhou et al., In vitro activity of cepharanthine hydrochloride against clinical wild-type and lamivudine-resistant hepatitis B virus isolates. Eur. J. Pharmacol. 683, 10-15 (2012).

52. C. Bailly, Cepharanthine: An update of its mode of action, pharmacological properties and medical applications. Phytomedicine 62, 152956 (2019). 
492

493

494

495

496

497

498

499

500

501

502

503

504

505

506

507

508

509

510

511

512

513

514

515

516

517

518

519

520

521

522

523

524

525

53. A. Fiorentini et al., The Time Has Come for Targeted Therapies for AML: Lights and Shadows. Oncol Ther 8, 13-32 (2020).

54. C. Müller et al., Comparison of broad-spectrum antiviral activities of the synthetic rocaglate CR-31-B (-) and the eIF4A-inhibitor Silvestrol. Antiviral Res. 175, 104706-104706 (2020).

55. A. I. Chiramel et al., Alisporivir Has Limited Antiviral Effects Against Ebola Virus Strains Makona and Mayinga. J. Infect. Dis. 214, S355-S359 (2016).

56. T. F. Rogers et al., Isolation of potent SARS-CoV-2 neutralizing antibodies and protection from disease in a small animal model. Science 369, 956-963 (2020).

57. S. Jäger et al., Global landscape of HIV-human protein complexes. Nature 481, 365-370 (2011).

58. D. Wang et al., A deep proteome and transcriptome abundance atlas of 29 healthy human tissues. Mol. Syst. Biol. 15, e8503 (2019).

59. A. Ruepp et al., CORUM: the comprehensive resource of mammalian protein complexes. Nucleic Acids Res 36, D646-D650 (2008).

60. N. Schmidt et al., The SARS-CoV-2 RNA-protein interactome in infected human cells. Nat. Microbiol., (2020).

61. R. A. Flynn et al., Discovery and functional interrogation of SARS-CoV-2 RNA-host protein interactions. Cell, (2021).

\section{Acknowledgments}

We thank members of the Zhang lab for discussion. We thank professor Jianbin Wang for the help of the project. We thank the Tsinghua University Branch of China National Center for Protein Sciences (Beijing) for the computational facility support.

Funding: This work is supported by the National Natural Science Foundation of China (Grants No. 31671355, 91740204, and 31761163007); National Key R\&D Program of China (2020YFA0707600), and Tsinghua-Cambridge Joint Research Initiative Fund. Author contributions: Q.C.Z. conceived the project. Q.C.Z., J.W. and Q.D. supervised the project. L.R. prepared the SARS-CoV-2 virus assisted by J.R.. S.J. performed the ChIRP-MS and validation experiments assisted by L.S.. X.J. developed the SARS-CoV-2 N trans-complementation system and screened the drugs. L.G. screened the drugs on EBOV. W.H. analyzed the ChIRP-MS data with the assisted by P.L.. Q.C.Z. and S.Z. wrote the manuscript with inputs from all authors. Competing interests: Authors declare no competing interests. 
527 Supplementary Materials

528 Materials and Methods

529 Data reporting

$530 \quad$ No statistical methods were used to predetermine sample size. The experiments 531 were not randomized and the investigators were not blinded to allocation during 532 experiments and outcome assessment.

\section{Cell line and antibodies}

535 Cell lines and antibodies used in this study are listed in (Table S6). All cell lines 536 were determined to be free of mycoplasma based on PCR and nuclear staining. All cell 537 lines mentioned in this study were cultured in DMEM (10\% FBS, $5381 \times$ Antibiotic-Antimycotic) under $37^{\circ} \mathrm{C}, 5 \% \mathrm{CO}_{2}$.

\section{$540 \quad$ Virus strains and cell infection}

541 For SARS-CoV-2 infection, Huh7.5.1 cells were cultured in T-175 flasks $(2 \times 3 \times 3$ 542 flasks, including 3 biological repeats, 3 flasks for each mock or infected ChIRP-MS), at 543 a density of $5 \times 10^{6}$ cells. The cells were briefly washed with DMEM at $16 \mathrm{~h}$ after

544 seeding, and incubated with a clinical isolate of SARS-CoV-2/IPBCAMS-YL01/2020

545 for $1 \mathrm{~h}$ at a multiplicity of infection (MOI) of 0.05 . Then the cells were supplemented 546 with DMEM maintenance medium containing $1 \% \mathrm{FBS}$ and cultured at $37^{\circ} \mathrm{C}, 5 \% \mathrm{CO}_{2}$ 547 for an additional $30 \mathrm{~h}$. The cultured cells were washed twice with PBS and 4\% 548 formaldehyde (Pierce, 28908) was added for crosslinking at room temperature for $4 \mathrm{~h}$. 549 Live virus was inactivated by an additional $12 \mathrm{~h}$ incubation with $4 \%$ formaldehyde at $5504{ }^{\circ} \mathrm{C}$. The cells were then collected and washed with $0.125 \mathrm{M}$ Glycine at room 551 temperature, followed by centrifugation at $1000 \mathrm{~g}, 4{ }^{\circ} \mathrm{C}$ for $5 \mathrm{~min}$ and 3 washes with 552 PBS. The cell pellets were used for ChIRP-MS experiments. Mock cells (no infection) 
553 were cultured and treated the same as the infected cells. All experiments involving live

554 SARS-CoV-2 in this study were performed in a biosafety level III facility.

555 For ZIKV infection, $7 \times 10^{6} \mathrm{Huh} 7$ cells were cultured on a $15-\mathrm{cm}$ dish for $20 \mathrm{~h}$

$556(2 \times 3 \times 3$ plates, including 3 biological repeats, 3 plates for each mock or infected

557 ChIRP-MS experiment), then infected with ZIKV (MR766, MOI 0.5). After $72 \mathrm{~h}$, cells

558 were collected using trypsin digestion and washed twice with cold PBS, followed by

559 crosslinking in PBS containing 3\% formaldehyde at room temperature for $30 \mathrm{~min}$.

560 Crosslinking was stopped by adding a $1 / 10$ volume of room temperature $1.25 \mathrm{M}$

561 Glycine for $5 \mathrm{~min}$. The cells were then washed with 3 times with PBS. After

562 centrifugation, PBS was removed and the cell pellets were used for ChIRP-MS. Mock

563 cells were cultured and treated like infected cells, but no virus was added.

564 EBOV $\triangle$ VP30-GFP(32) (strain Zaire Mayinga) virions were generated using VP30 565 expressing Vero E6 cell lines, then infected Huh7.5.1 cells expressing VP30 566 (Huh7.5.1-VP30) at a MOI=0.1. For EBOV ChIRP-MS experiment, Huh7.5.1-VP30 567 cells were cultured on $15-\mathrm{cm}$ plates $(2 \times 3 \times 2$ plates, including 2 biological repeats, 3 568 plates for each mock or infected ChIRP-MS) at a density of $6 \times 10^{6}$ cells, then infected 569 with EBOV $\triangle$ VP30-GFP virus. After $72 \mathrm{~h}$, infected and mock cells were collected and 570 crosslinked as in the ZIKV experiment.

571

\section{Identifying host proteins interacting with viral RNA by ChIRP-MS}

573 ChIRP-MS was performed according to the previous report(19) with some 574 modifications. Briefly, crosslinked cells were resuspended in $1 \mathrm{~mL}$ lysis buffer 575 ( 100mg cells) containing 50 $\square \mathrm{mM}$ Tris-HCl, pH $\square 7.0,10 \square \mathrm{mM}$ EDTA, $1 \%$ SDS, $0.1 \%$

576 sodium deoxycholate, $0.5 \%$ DDM and $0.1 \%$ NP-40) and sonicated. Probes ( $2 \mathrm{uL}$ of 100

$577 \mu \mathrm{M}$ ) tiling the whole genome of virus (Table S1) were used to capture vRNA-protein 578 complexes. MyOne C1 beads (Invitrogen,65001) were blocked with 3\% BSA and yeast 579 tRNA (Solarbio, T8630), and $200 \mathrm{uL}$ of $\mathrm{C} 1$ beads was used for each ChIRP experiment.

580 The captured materials were firstly washed with lysis buffer for 2 times $\times 5 \mathrm{~min}$, then 
581 washed with ChIRP wash buffer $(2 \times \mathrm{SSC}, 0.5 \% \mathrm{SDS})$ for 4 times $\times 5 \mathrm{~min}$. The

582 co-purified proteins were reverse crosslinked using $0.3 \mathrm{M} \mathrm{NaCl}$ buffer $(7.5 \square \mathrm{mM}$

583 HEPES, pH $\square 7.9,12.5 \square \mathrm{mM}$ D-biotin, $0.3 \mathrm{M} \mathrm{NaCl}, 1.5 \square \mathrm{mM}$ EDTA, $0.2 \%$ SDS, $5 \mathrm{mM}$

584 DTT, $0.075 \%$ sarkosyl and $0.02 \%$ sodium deoxycholate) at $70{ }^{\circ} \mathrm{C}$ with shaking for $1 \mathrm{~h}$.

585 Proteins were precipitated in $20 \%$ TCA, then the protein precipitate was resuspended in

586 RIPA buffer (25mM Tris-HCl, pH 7.4, 1\% NP-40, 0.5\% DOC, 0.1\% SDS). Protein

587 samples were loaded onto an SDS-PAGE gel and visualized using silver staining.

588 Protein bands were excised from the gel and de-stained following product instructions

589 (Pierce Silver Stain for Mass Spectrometry, 24600). The excised gel bands were

590 desalted, $\mathrm{pH}$ adjusted, and digested using Trypsin overnight. The digested peptides

591 were extracted from the gel using $50 \%$ acetonitrile and $0.1 \%$ formic acid, and analyzed

592 via mass spectrometry (Thermo Scientific Q Exactive). ChIRP-MS control

593 experiments were performed on cells without viral infection (mock) by using the same

594 set of probes.

595 To determine the viral RNA recovery rate for ChIRP-MS (\%RNA retrieve), $10 \mu \mathrm{L}$

596 of lysate $(1 \%)$ was removed from $1 \mathrm{~mL}$ of sonicated cell lysate, and used as input

597 sample. At the last wash, a $10 \mu \mathrm{L}$ volume of wash buffer containing beads (1\%) was

598 removed as the eluate for RNA quantification. Samples (input and eluate) were

599 suspended in $100 \mu \mathrm{L}$ PK buffer (10 mM Tris-HCl pH 7.0, $100 \mathrm{mM} \mathrm{NaCl}, 0.5 \%$ SDS, 1

$600 \mathrm{mM}$ EDTA) containing $20 \mu \mathrm{g} / \mathrm{mL}$ proteinase $\mathrm{K}$ (Roche, 3115879001). The input and

601 eluate samples were incubated at $50{ }^{\circ} \mathrm{C}$ for $45 \mathrm{~min}$ and then $95{ }^{\circ} \mathrm{C}$ for $10 \mathrm{~min}$ with

602 mixing. Finally, $300 \mu \mathrm{L}$ of TRIzol LS reagent was added to extract RNA following the

603 manufacturer's instructions. For both input and eluate, $2 \mu \mathrm{L}$ of RNA was used for

604 reverse transcription for cDNA synthesis using a PrimeScript RT reagent Kit

605 (TAKARA, RR047A). For EBOV, sequence specific primers for the EBOV genome

606 and Gapdh mRNA were used for reverse transcription. $q$ PCR of viral RNA and Gapdh

607 were performed using SYBR Green kits (TAKARA, RR420A) following the 
608 manufacturer's instructions. The following primers were used for reverse transcription

609 or $q \mathrm{PCR}$ :

610 Gapdh Forward primer: ACACCCACTCCTCCACCTTTGAC;

611 Gapdh Reverse primer: ACCCTGTTGCTGTAGCCAAATTC.

612 ZIKV E Forward primer: CCGCTGCCCAACACAAGGTGAAG;

613 ZIKV E Reverse primer: CCACTAACGTTCTTTTGCAGACAT.

614 SARS-CoV-2 NP Forward primer: GGGGAACTTCTCCTGCTAGAAT;

615 SARS-CoV-2 NP Reverse primer: CAGACATTTTGCTCTCAAGCTG.

616 EBOV NP Forward primer: CCGTTCAACAGGGGATTGTTCG;

617 EBOV NP Reverse primer: CTGCTGGCAGCAATTCCTCAAG.

618 EBOV reverse transcription primer: CTCAGAAAATCTGGATGGCGCCGAGTCTC

619 Gapdh reverse transcription prime: CTGAGTGTGGCAGGGACTCCCCAG

620

621 ChIRP-WB

622 For ChIRP-western blotting (ChIRP-WB), Huh7.5.1 (SARS-CoV-2), 623 Huh7.5.1-VP30 (EBOV), or Huh7 (ZIKV) cells were cultured on ten 10-cm plates, 624 infected with virus and crosslinked as above described. Then, $\sim 150 \mathrm{mg}$ mock or 625 infected cells were resuspended in $1 \mathrm{~mL}$ lysis buffer and sonicated. ChIRP experiments 626 were performed as described above. $10 \mathrm{uL}$ of cell lysate (per $1 \mathrm{~mL}, 1 \%$ ) was removed 627 as input. After washing, MyOne $\mathrm{C} 1$ beads were resuspended using $50 \mathrm{uL}$ of $0.3 \mathrm{M} \mathrm{NaCl}$ 628 elution buffer (7.5 $\square \mathrm{mM}$ HEPES, pH $\square 7.9,12.5 \square \mathrm{mM}$ D-biotin, $0.3 \mathrm{M} \mathrm{NaCl}, 1.5 \square \mathrm{mM}$ 629 EDTA, $0.2 \%$ SDS, 5mM DTT, 0.075\% sarkosyl and 0.02\% sodium deoxycholate), 630 boiled at $70{ }^{\circ} \mathrm{C}$ for $1 \mathrm{~h}$ and then at $95{ }^{\circ} \mathrm{C}$ for $30 \mathrm{~min}$ to elute proteins. $10 \mathrm{uL}$ of the 631 protein eluate and input samples were separated on SDS-PAGE gels and 632 immunoblotted using the specific antibodies listed in Table S6. 


\section{ChIRP-MS data analysis}

635 Raw mass spectrometry data were processed with Proteome Discover using the 636 built-in search engine to search against the human proteome (Uniprot database). Viral 637 proteins, including SARS-CoV-2 (NC_045512.2), ZIKV (NC_012532.1), and EBOV 638 (NC_002549.1) proteins were downloaded from NCBI and added into the database 639 manually. Proteomic data were filtered by applying a minimum Protein Score of 1.5.

640 Viral RNA interacting proteins were scored with the MiST scoring algorithm(57) using 641 default parameters. For SARS-CoV-2 and EBOV infected samples, proteins identified 642 with a MiST score $>0.6$ were considered to interact with viral RNA. For ZIKV infected 643 samples, the cutoff MiST score was set as > 0.7. Every identified protein was assessed 644 with the same parameters as in MiST, i.e., "Abundance" to represent protein abundance 645 in virus infected samples, defined as the mean of the bait-prey quantities (spectral 646 counts divided by protein length) over all replicates for virus-infected samples; 647 "Specificity" to measure the uniqueness of proteins identified in virus-infected samples 648 compared with non-infected samples (mock), defined as the proportion of the 649 abundance of virus infected samples compared to the abundance of non-infection 650 samples; "Reproducibility" to evaluate the variance of each identified protein 651 abundance among replicates, defined as the normalized entropy of the bait-prey 652 quantities over all replicates for virus-infected samples. The proteins interacting with at 653 least two viral RNAs were defined as common interacting proteins.

654

\section{Differential protein expression analysis}

656 To analyze differential protein expression of SARS-CoV-2 vRNA interacting 657 proteins $(n=143)$ across human tissues, we obtained protein abundance values in 29 658 human tissues from a proteomics dataset(58). Then the abundances of interacting 659 proteins in each tissue were compared to their medians protein abundance among all 29

660 tissues. The significance $P$ value of enrichment was calculated by Mann-Whitney U 661 test and adjusted by FDR. 


\section{Protein domain analysis}

664 To analyze whether there were possible enriched protein domains among the 665 identified proteins, we annotated the domains of identified proteins using the Uniprot 666 database. For each protein domain, we counted the number of identified proteins 667 containing this domain. The enrichment (odds ratio) and significance $P$ value of each 668 domain among identified proteins, compared to all human proteins, was calculated by 669 Fisher's exact test.

$$
\text { odds ratio }=\frac{\frac{\text { number of identified proteins containing the domain }}{\text { number of identified proteins not containing the domain }}}{\frac{\text { number of all proteins containing the domain }}{\text { number of all proteins not containing the domain }}}
$$

670

\section{Protein complex analysis}

672 To analyze whether there were possible enriched protein complexes among the 673 identified proteins, we performed a protein complex enrichment analysis using the 674 CORUM database(59). For each protein complex, we counted the number of identified 675 proteins in this complex. The enrichment (odd ratio) and significance $P$ value of these 676 proteins in each complex, compared to all human proteins, was calculated by Fisher's 677 exact test.

$$
\text { odds ratio }=\frac{\frac{\text { number of identified proteins in complex }}{\text { number of identified proteins not in complex }}}{\frac{\text { number of all proteins in complex }}{\text { number of all proteins not in complex }}}
$$

678 We searched the significantly enriched complexes $(P$ value $<0.05)$ for each of the 679 three viral RNA interactomes. Protein complexes present in at least two of the three 680 virus interactomes were defined as common interacting complexes. Complexes present 681 in only a single virus interactome were defined as virus-specific interacting complexes. 682 


\section{GO and KEGG enrichment analyses}

684 We performed gene ontology (GO) and KEGG pathway enrichment analyses of 685 the identified proteins using The Database for Annotation, Visualization and Integrated 686 Discovery (DAVID) v6.8. The significance $P$ values of GO terms and KEGG pathways 687 were calculated by Fisher's exact test. Top 10 enriched GO terms or KEGG pathways $688 \quad(P$ value $<0.05)$ were shown.

689

690 Interactome-informed drug discovery

691 SARS-CoV-2 host factors were assessed looking for drugs known to impact 692 human proteins. Briefly, to identify drugs/compounds modulating 143 human 693 interacting proteins, protein uniport IDs were searched against the IUPHAR/BPS 694 Guide to Pharmacology, Drugbank, Drugcentral, and ChEMBL databases. Then we 695 retrieved 5,309 compounds from these databases related to 56 host factors (Table S4). 696 Specifically, 7 compounds related with 5 interacting proteins from the IUPHAR/BPS 697 Guide to Pharmacology; 158 compounds related with 44 interacting proteins from the 698 Drugbank; 19 compounds related with 6 interacting proteins from the Drugcentral and 6995125 compounds predicted to be related with 35 interacting proteins from the ChEMBL 700 (Table S4). Retrieved molecules were prioritized based on their FDA approval status 701 and commercial availability. FDA approved drugs were prioritized for testing its 702 antiviral activities; an exception is Silvestrol, which is an eIF4A specific inhibitor 703 currently under investigation in a registered preclinical trial.

\section{Antiviral drug screening}

706 Based on the SARS-CoV-2 reference sequence (Wuhan-Hu-1, NC_045512), we 707 cloned the SARS-CoV-2 genome as five fragments. These fragments were then 708 assembled using in vitro ligation but replacing the viral $\mathrm{N}$ gene with GFP, to generate 709 the SARS-CoV-2-GFP $\Delta \mathrm{N}$ genome. Genomic RNA of SARS-CoV-2-GFP $\Delta \mathrm{N}$ and 710 mRNA of $\mathrm{N}$ gene were in vitro transcribed using an mMESSAGE mMACHINE T7 
711 Transcription Kit (Thermo Fisher Scientific, AM1344). Synthesized viral RNA was

712 electroporated into Vero E6-N cells that were stably expressing the SARS-CoV-2 N

713 protein generated by lentivirus transduction, to produce the $\mathrm{P}_{0}$ virus. After $72 \mathrm{~h}, \mathrm{P}_{0}$

714 virus was collected. Caco2 cells stably expressing the $\mathrm{N}$ protein (Caco2-N) were

715 generated using lentivirus infection; this Caco2-N cell line can support the infection 716 and replication of the SARS-CoV-2-GFP $\triangle \mathrm{N}$ virus.

717 To screen compounds with antiviral effects, Caco2-N cells were cultured on 718 96-well plates at a density of $1 \times 10^{4}$ cells per well. After $24 \mathrm{~h}$, cells were infected with 719 SARS-CoV-2-GFP $\triangle \mathrm{N}$ virus at a MOI of 0.05 and analyte drugs were administered at 720 the same time as the virus. After $72 \mathrm{~h}$, FACS was performed to quantify 721 GFP-expressing cells. Remdesivir was used as a positive antiviral control. The 722 infection ratios resulting from treatment with various drug concentrations were 723 normalized to the DMSO $(0.2 \%)$ treated cells.

724 Compounds, known to target common interacting proteins and which showed 725 antiviral effects against SARS-CoV-2-GFP $\triangle \mathrm{N}$ were also tested for EBOV and ZIKV 726 infection. To assess the antiviral effects of the compounds on EBOV, Huh7.5.1-VP30 727 cells were cultured on 96 well plates with $1 \times 10^{4}$ cells per well. After $20 \mathrm{~h}$, drugs were 728 added and cells were infected at the same time with EBOV $\Delta$ VP30-GFP virus (strain 729 Zaire Mayinga) at a MOI of 0.1. After $72 \mathrm{~h}$, GFP-expressing cells were counted using 730 FACS. Remdesivir was used as a positive antiviral control. The infection ratios with 731 various drug concentrations were normalized to the DMSO $(0.4 \%)$ treated cells.

732 For ZIKV, Huh7 cells were cultured on 96 well plates (Corning, 3603) with $1 \times 10^{4}$ 733 cells per well. To test the antiviral effects of drugs, Huh7 cells were infected with ZIKV 734 (MR766) at a MOI of 0.5 and drugs were added at the same time. At $72 \mathrm{~h}$, cells were 735 fixed using 4\% PFA, and blocked using blocking buffer (1×PBS, 5\% FBS, $0.3 \%$ Triton 736 X-100). The fixed cells were then incubated with Anti-Flavivirus Group Antigen 737 Antibody (clone 4G2, MAB10216, 1:1000) in blocking buffer for $12 \mathrm{~h}$ at $4{ }^{\circ} \mathrm{C}$. After 738 washing 3 times with PBS, secondary antibody (Goat Anti-Mouse IgG H\&L Alexa 
739 Fluor 488, ab150113) was diluted in blocking buffer (1:1000) containing DAPI (5

$740 \mathrm{ug} / \mathrm{mL}$ ) and was added and incubated for $2 \mathrm{~h}$ at room temperature. The ZIKV infection

741 ratio was then quantified using an Opera Phenix High-content System. Total cell

742 numbers were quantified based on DAPI staining of nuclei, and infected cells were

743 quantified according the Alexa Fluor 488 staining in cytoplasm. The infection ratio was

744 calculated as the number of Alexa Fluor 488 stained cells divided by the number of

745 DAPI stained nuclei. The infection ratios with various drug concentrations were

746 normalized to the DMSO $(0.4 \%)$ treated cells.

747 For cell viability assays, Caco2-N, Huh7.5.1-VP30, and Huh7 were seeded on 96

748 well plates with $1 \times 10^{4}$ cells per well, and treated using drugs with different

749 concentrations. At 72 h, cell viability was measured using CellTiter-Glo Luminescent

750 Cell Viability Assay kits (Promega, G7570). Cell viability values were normalized to

751 DMSO treated cells (0.2\% for Caco2-N, 0.4\% for Huh7 and Huh7.5.1-VP30 cells).

752 Viral infection curves and cell viability curves were fitted using GraphPad Prism 8

753 (Nonlinear regression, Dose-response-Inhibition); the half maximal inhibitory

754 concentration (IC50) and the half maximal cytotoxic concentration (CC50) values were

755 calculated. The selective index (SI) was also calculated as the ratio of CC50/IC50.

756

\section{Supplementary Tables}

758 Table S1 Oligo probes used for ChIRP-MS

759 Table S2 Viral RNA interacting proteins identified by ChIRP-MS

760 Table S3 Enriched complexes of viral RNA interacting proteins

761 Table S4 The information of drugs targeting SARS-CoV-2 RNA interacting human

762 proteins from drug database

763 Table S5 Information of the 21 selected drugs

764 Table S6 Regents, cell line, virus and antibodies

765

766 


\section{$767 \quad$ Figures and Figure Legends}

Fig. 1. Identification of the human proteins interacting with the SARS-CoV-2

\section{$770 \quad$ RNA genome in infected cells.}

771 A, Schematic for identifying host proteins that interact with viral RNAs using 772 ChIRP-MS. FA, formaldehyde. ZIKV, zika virus. EBOV, Ebola virus. Human cells 773 were infected with SARS-CoV-2, ZIKV or EBOV. Cells without infection (Mock)

774 were used for control. Infected and mock cells were crosslinked using formaldehyde, 775 and then sonicated to release RNA-protein complexes. For each virus, the viral RNA 776 (vRNA)-human protein complexes were purified using biotinylated oligos specifically

777 tiling the viral RNA genome, and the co-purified human protein were identified using 778 mass spectrometry.

779 B, Evaluation of the SARS-CoV-2 vRNA interacting proteins identified by 780 ChIRP-MS. Peak intensities of peptides of a protein from the mass spectrum 781 (Abundance), protein abundance over different replicates (Reproducibility), and 782 uniqueness of an interacting protein across all replicated experiments (Specificity) 783 were evaluated to define viral RNA interacting proteins (see Materials and Methods,

784 ChIRP-MS data analysis).

785 C, Correlation of $\log _{10}$ (PSMs) for proteins from three biological replicates.

786 In $\mathbf{B}$ and $\mathbf{C}$, interacting human proteins are indicated as red dots if also known to 787 interact with other coronavirus genome RNA, and otherwise as orange dots; 788 SARS-CoV-2 viral proteins co-purified with viral RNA are blue dots.

789 D, Validation of the interactions between the SARS-CoV-2 vRNA and human 790 proteins using ChIRP-Western blotting (ChIRP-WB). GAPDH protein, which is not 791 known to bind SARS-CoV-2 vRNA, was used as a negative control for ChIRP-WB 792 experiments. Mock, cells without infection. Infec., cells infected with SARS-CoV-2. 793 *, indicates proteins that are not consistent between ChIRP-MS and ChIRP-WB. 
794 E, Distribution of enriched protein domains among the interacting human proteins of

795 SARS-CoV-2 vRNA. Bold lines with different colors represent different protein

796 domains. Protein domains not known as RNA binding domains are indicated using

797 grey dashed boxes.

798 F, Human tissues or organs with significantly elevated expression levels for 799 SARS-CoV-2 vRNA interacting proteins $(\mathrm{n}=143) . P$ value was calculated by 800 Mann-Whitney U test and adjusted by FDR. The 10 tissues with the most significant $801 \quad P$ values are shown.

802 G, SARS-CoV-2 vRNA interacting proteins are more abundant than other 803 non-interacting proteins in lungs. $P$ value was calculated by Mann-Whitney $U$ test 804 and adjusted by FDR.

$805 \mathbf{H}$, Gene Ontology enrichment analysis of the SARS-CoV-2 vRNA interacting 806 proteins. The top 10 enriched terms are shown.

807 I, Cellular pathway (KEGG) enrichment analysis of the SARS-CoV-2 vRNA 808 interacting proteins. The top 10 enriched terms are shown.

809

810 Fig. 2. ChIRP-MS identified the human proteins interacting with the ZIKV and 811 EBOV RNA genomes in infected cells.

812 A, B, Evaluation of the ZIKV (a) or EBOV (b) vRNA interacting proteins identified

813 by ChIRP-MS. Definitions of "specificity", "abundance" and "reproducibility" are the 814 same as Fig. 1B (see Materials and Methods, ChIRP-MS data analysis). Host proteins 815 interacting with the ZIKV or EBOV vRNA are indicated using orange dots. ZIKV or 816 EBOV proteins co-purified with viral RNA are indicated as blue dots.

817 C, Validation of interactions between the indicated proteins with ZIKV or EBOV 818 vRNA using ChIRP-WB. HIST1H3A was used as a negative control. Mock, cells 819 without infection. ZIKV or EBOV, cells infected with indicated virus. *, indicates 820 proteins that are not consistent between ChIRP-MS and ChIRP-WB. 
821 D, Comparison of common interacting proteins between the viral RNA interactomes 822 for SARS-CoV-2, EBOV, and ZIKV.

823 E, Distribution of enriched protein domains among the common (left) and total (right) 824 interacting human proteins of SARS-CoV-2, ZIKV, and EBOV vRNA. Bold lines

825 with different colors represent different protein domains. Protein domains not known

826 as RNA binding domains are indicated using grey dashed boxes.

827 F, Gene Ontology enrichment analysis of the common (left) and total (right) 828 interacting human proteins of SARS-CoV-2, ZIKV, and EBOV vRNA. The top 10 829 enriched terms are shown.

830

831 Fig. 3. A compendium of viral RNA-human protein interactomes for 832 SARS-CoV-2, EBOV, and ZIKV.

833 Central nodes represent viral RNAs. Circular nodes represent host proteins. Edges

834 indicate RNA-protein interactions (RPI, orange) and protein-protein interactions (PPI,

835 blue). Protein complexes based on the CORUM database and proteins associated with

836 the same biological processes according to functional annotation are enclosed with

837 dashed lines. Nodes are colored according to the MiST scores of proteins interacting

838 with SARS-CoV-2 (orange), ZIKV (blue), and EBOV (green) vRNA. Drugs targeting

839 or predicted to target the indicated proteins (Table S5, Materials and Methods,

840 Interactome-informed drug discovery) are highlighted with red circles.

841

842 Fig. 4. Validation of antiviral activities of the FDA-approved and clinical-trial 843 drugs targeting viral RNA interacting proteins.

844 A, FDA-approved (green) and clinical-trial (black) compounds modulating the 845 SARS-CoV-2 vRNA interacting proteins are presented. Nodes are colored by the 846 MiST scores of SARS-CoV-2 vRNA interacting proteins, and protein names are 847 colored by the up- (red) or down-regulation upon SARS-CoV-2 infection(27). 
848 B, Antiviral activity test for the selected drugs using a SARS-CoV-2-GFP $\Delta \mathrm{N}$

849 trans-complementation system. For this validation system, the SARS-CoV-2 genome

850 sequence segment encoding nucleocapsid (N) was replaced by GFP, thus the resulted

851 SARS-CoV-2-GFP $\Delta \mathrm{N}$ only amplifies in $\mathrm{N}$ protein-expressing Caco-2 (Caco-2-N)

852 cells. The infection ratio was then evaluated by quantifying the GFP positive cells.

853 For initial testing of antiviral activities, drugs were added at the same time as the

854 infection (MOI=0.05), at a concentration of $10 \mu \mathrm{M}$. GFP ratios (indicating the

855 infection ratio of SARS-CoV-2) were calculated based on FACS data after $72 \mathrm{~h}$.

856 Remdesivir was used as antiviral positive control.

857 C, SARS-CoV-2-GFP $\triangle \mathrm{N}$ infection ratios in cells treated with the indicated drugs at a 858 concentration of $10 \mu \mathrm{M}$. Caco2-N cells were infected with SARS-CoV-2-GFP $\Delta \mathrm{N}$

859 virus for $72 \mathrm{~h}$ with drug treatment at day 0. Black bar, infection ratios of the

860 SARS-CoV-2-GFP $\triangle N$. Grey bar, cell viabilities after treatment with the indicated

861 compounds at $10 \mu \mathrm{M}$. Data were normalized to DMSO treatment. Remdesivir as a

862 positive control $(2 \mu \mathrm{M})$. Drugs selected for further validation were highlighted in red.

863 Drugs with modest antiviral effects were highlighted in yellow. Drugs with no

864 significant antiviral activities at a concentration of $10 \mu \mathrm{M}$ were in black. Data are

865 means \pm SD. $\mathrm{n}=4$ biologically independent samples. $* *<0.01, *<0.05$, Student's

$866 t$-test.

867 D-F, The antiviral activities of Silvestrol, CsA, Cepharanthine, Trifluoperazine, and

868 Enasidenib against SARS-CoV-2 (D), ZIKV (E), and EBOVAVP30-GFP (F)

869 infections at different drug concentrations. Red line, cell viability; Black line,

870 infection ratio relative to DMSO treatment. Data are means \pm SD. $n=3$ biologically

871 independent samples. IC50, CC50, and SI values are indicated.

872 


\section{Supplementary Figures}

874 Fig. S1. Identification of the human proteins interacting with SARS-CoV-2 875 vRNA in infected cells.

876 A, SARS-CoV-2 vRNA recovery rates of ChIRP-MS experiments. $q$ PCR of viral 877 RNA (NP gene) was performed in ChIRP input and elution samples, Gapdh was used 878 as a SARS-CoV-2 probe non-targeting control. Data are means $\pm \mathrm{SD}, \mathrm{n}=3$ 879 biologically independent samples of ChIRP-MS.

880 B, Protein domain enrichment analysis of the interacting proteins of SARS-CoV-2 881 vRNA. The top 10 enriched terms are shown.

882 C, Comparison of SARS-CoV-2 vRNA interacting proteins identified in this study 883 with protein groups from two different RNA pull-down method (60, 61) (Fisher's 884 exact test).

885

886 Fig. S2. Identification of human proteins interacting with ZIKV and EBOV 887 vRNAs in infected cells.

888 A, Viral RNA retrieved by ChIRP-MS experiments. qPCR of viral RNA (ZIKV E 889 protein gene and EBOV NP gene) was performed in ChIRP-MS input and elution 890 samples, Gapdh mRNA was used as a non-targeting control for biotinylated oligo 891 probes. Data are means $\pm \mathrm{SD}, \mathrm{n}=3$ biologically independent samples for ZIKV, $\mathrm{n}=2$ 892 biologically independent samples for EBOV ChIRP-MS, respectively.

$893 \mathbf{B}, \mathbf{C}$, Correlation of $\log _{10}$ (PSMs) for proteins identified in ZIKV infected Huh7 cells

894 (B) and EBOVAVP30-GFP infected Huh7.5.1-VP30 cells (C), respectively. Data 895 present Pearson correlation coefficient of $\log _{10}$ (PSMs) from biological replicates. The 896 viral RNA interacting proteins are indicated using red dots in $\mathbf{B}$ and $\mathbf{C}$; Viral proteins 897 co-purified with viral RNA are in blue. 
898 D, ZIKV vRNA interacting proteins identified by different RNA pull-down methods.

899 Human protein groups identified in the present study was compared with the protein

900 groups identified by other studies(17).

901 Fig. S3. Functional analysis of the viral RNA-specific interacting proteins.

902 A, B, Gene Ontology (A) and KEGG pathway (B) enrichment analysis of proteins 903 specifically interacting with the SARS-CoV-2 vRNA. The top 10 enriched terms are 904 shown.

905 C, D, Gene Ontology (C) and KEGG pathway (D) enrichment analysis of proteins 906 specifically interacting with the ZIKV vRNA. The top 10 enriched terms are shown.

907 E, F, Gene Ontology (E) and KEGG pathway (F) enrichment analysis of proteins 908 specifically interacting with the EBOV vRNA. The top 10 enriched terms are shown. 909 
Fig. 1

bioRxiv preprint doi: https://doi.org/10.1101/2021.04.25.441316; this version posted April 26, 2021. The copyright holder for this preprint (which A

was not certified by peer review) is the author/funder. All rights reserved. No reuse allowed without permission.

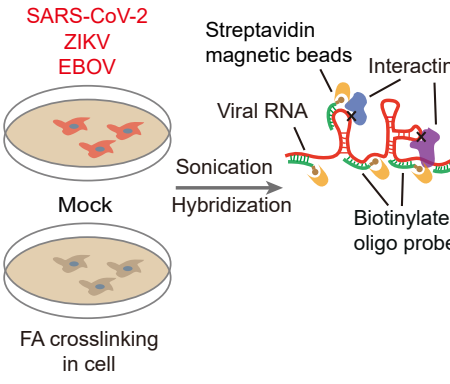

B

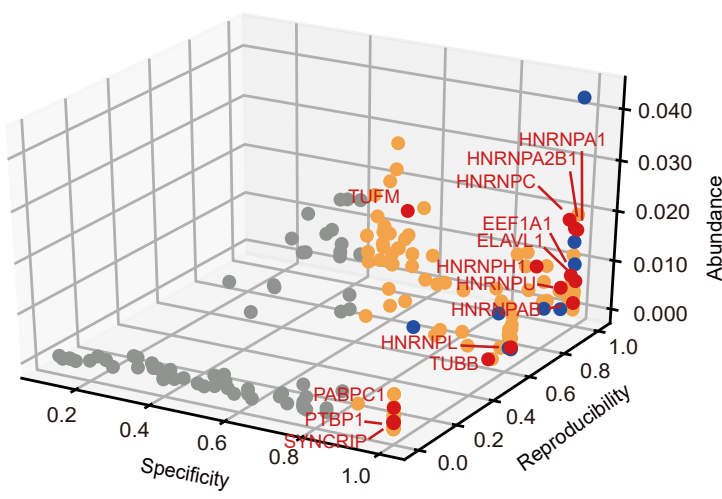

- Proteins interacting with SARS-CoV-2 and other coronavirus VRNA

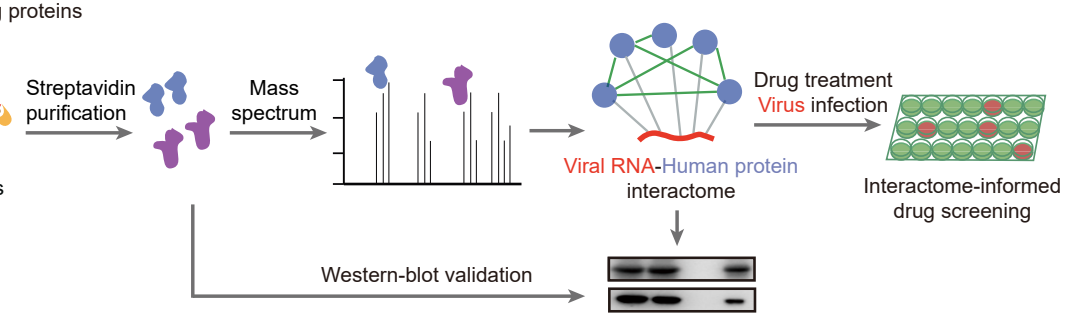

C

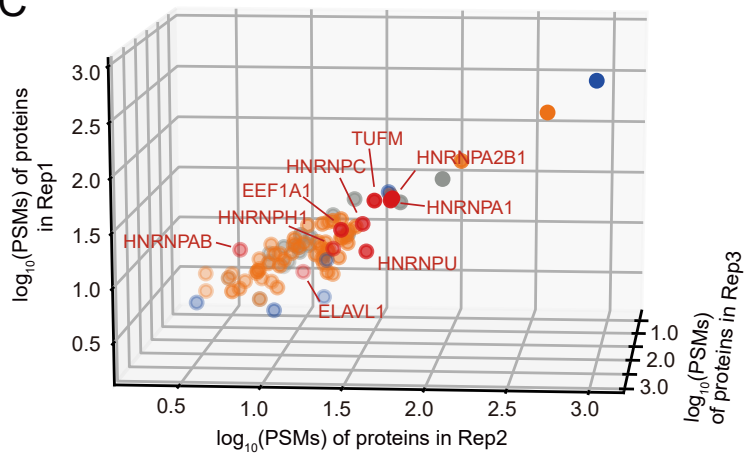

- Protein interacting with SARS-CoV-2 vRNA
- Viral protein
D
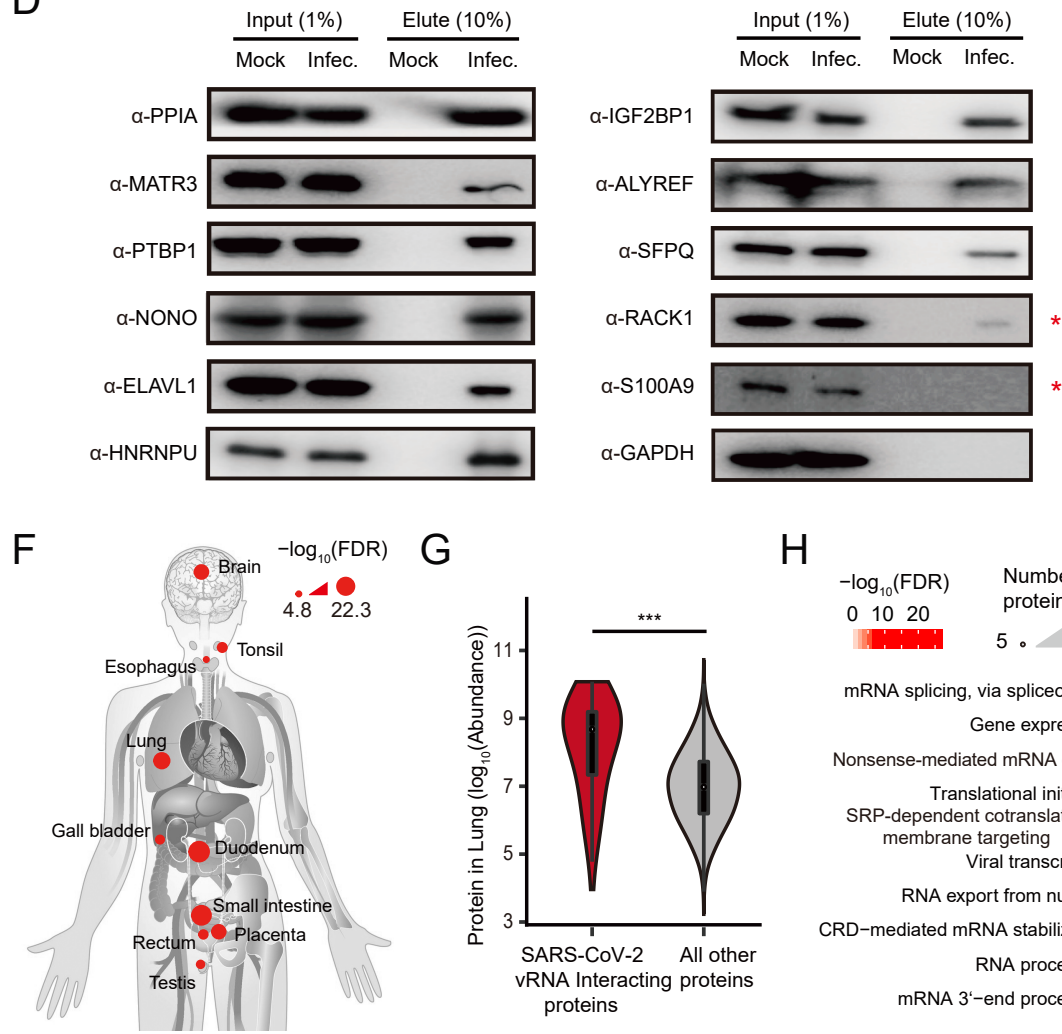

$\mathrm{H}$

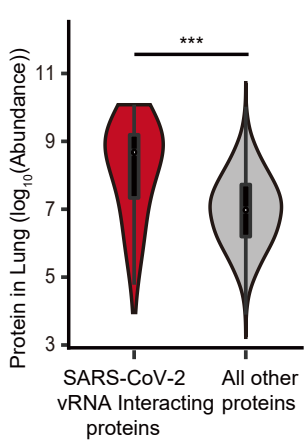

$E$

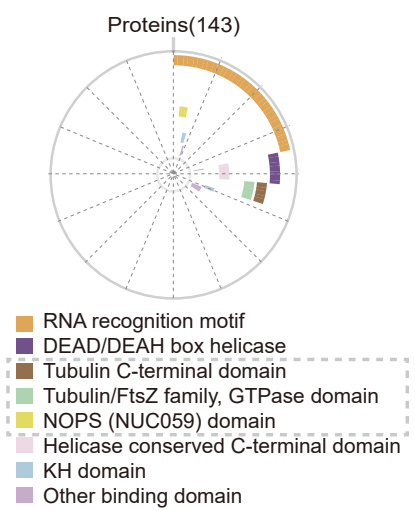

Other binding domain 


\section{Fig. 2}

bioRxiv preprint doi: https://doi.org/10.1101/2021.04.25.441316; this version posted April 26, 2021. The copyright holder for this preprint (which

A

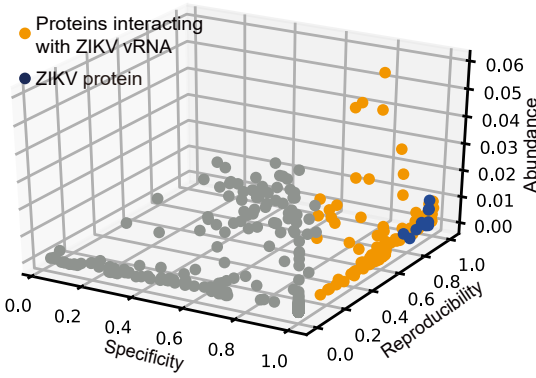

was not certified by peer review) is the author/funder. All rights reserved. No reuse allowed without permission.

$\mathrm{B}$

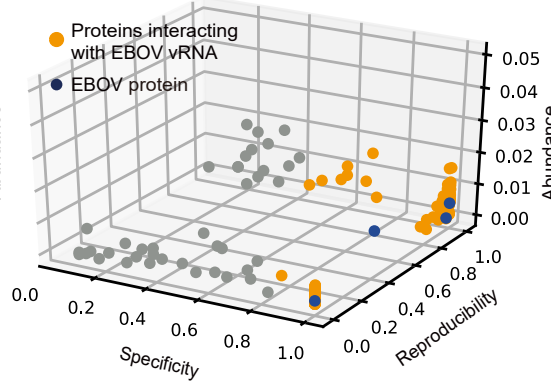

C

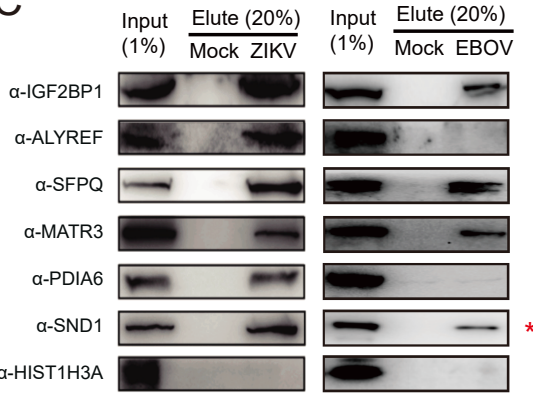

$\mathrm{D}$

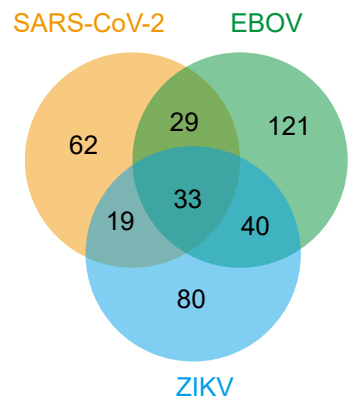

E

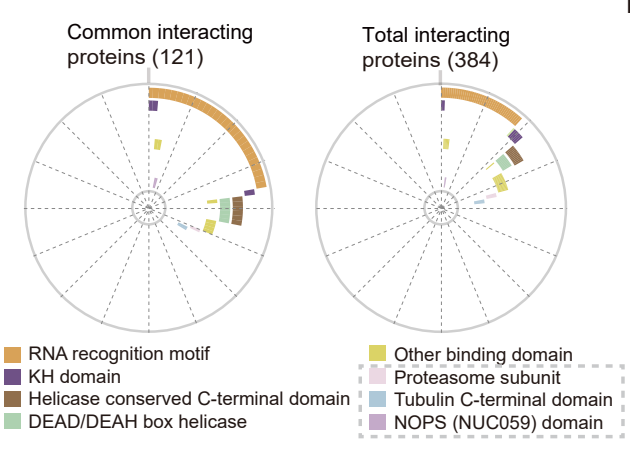

$\mathrm{F}$

$-\log _{10}($ FDR $) \quad$ Number of Common Total

02040 proteins found interacting interactin

SRP-dependent cotranslational

membrane targeting
Nonsense-mediated mRNA decay

Translational initiation

Viral transcription $\bullet \quad 0$

rRNA processing $\circ$

Translation $\circ \bigcirc$

mRNA splicing, via spliceosome $\quad$ ○

$\begin{array}{ccc}\text { Gene expression } & \circ & \circ \\ \text { Cell-cell adhesion } & \circ & \circ\end{array}$

Negative regulation of translation . . 
Fig. 3

bioRxiv preprint doi: https://doi.org/10.1101/2021.04.25.441316; this version posted April 26, 2021. The copyright holder for this preprint (which was not certified by peer review) is the author/funder. All rights reserved. No reuse allowed without permission.

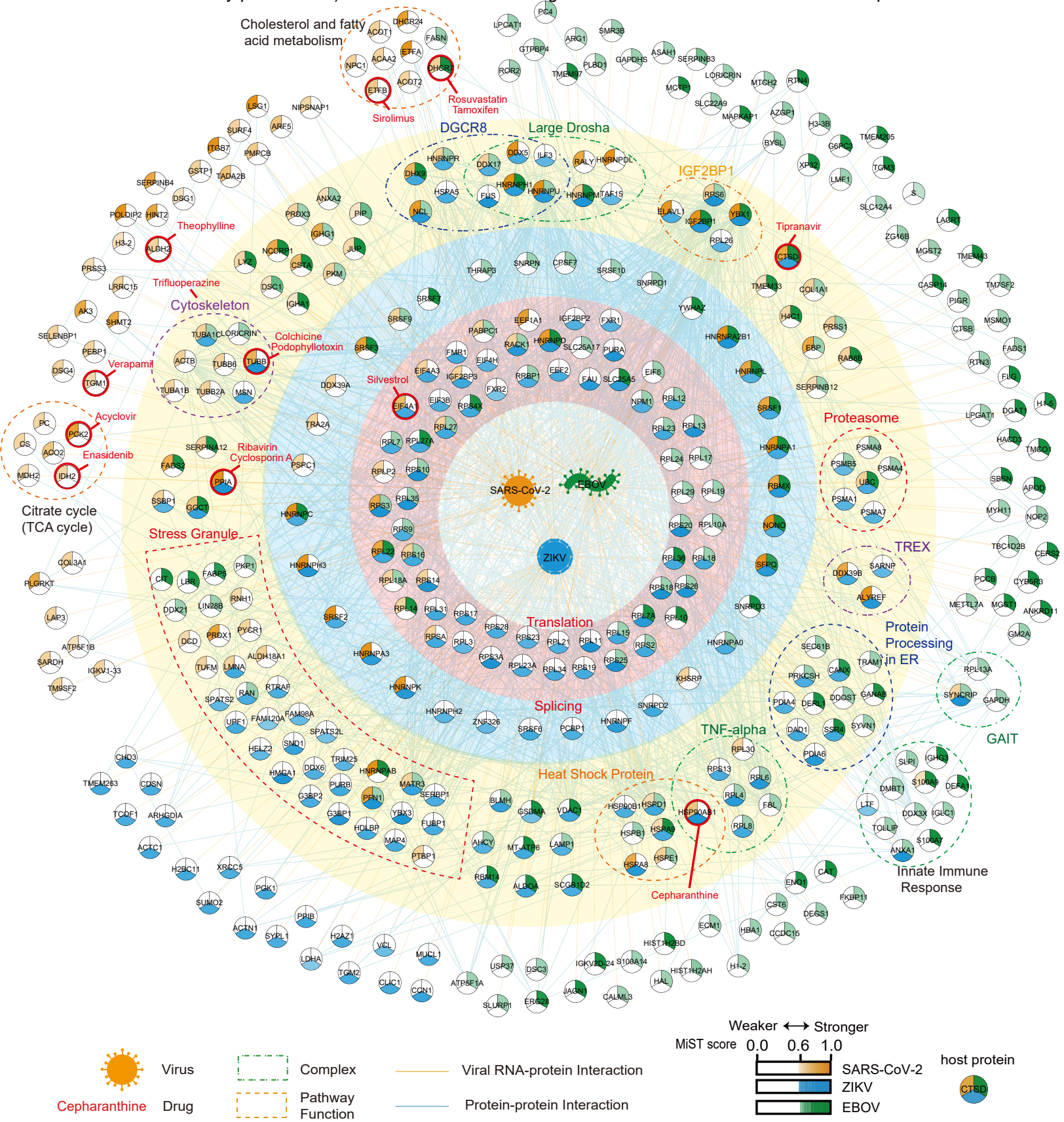


Fig 4

A
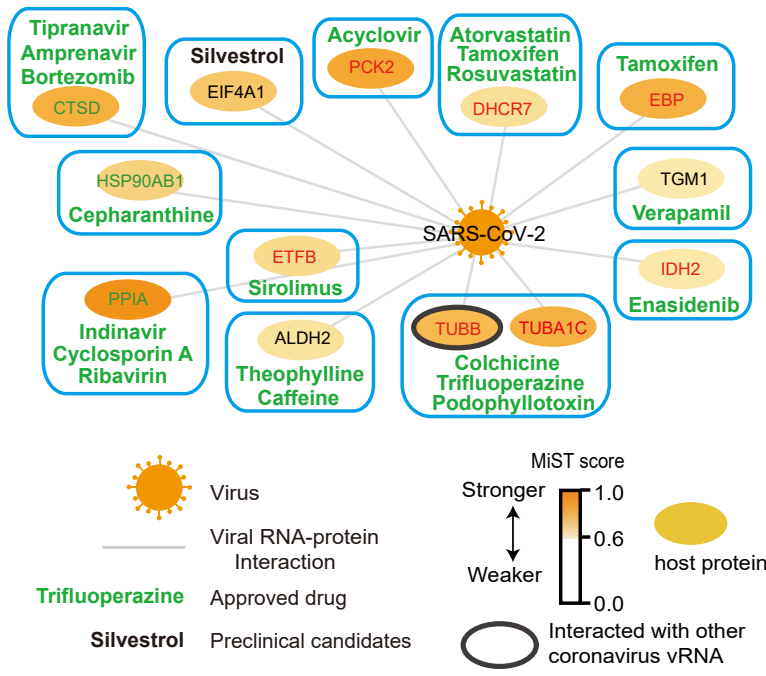

C

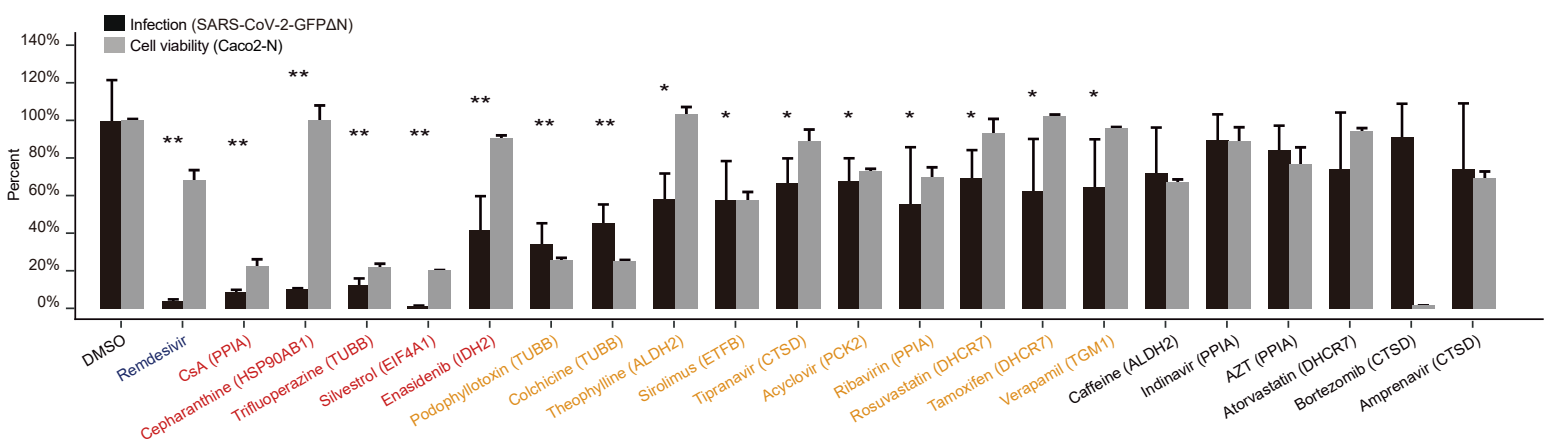

D
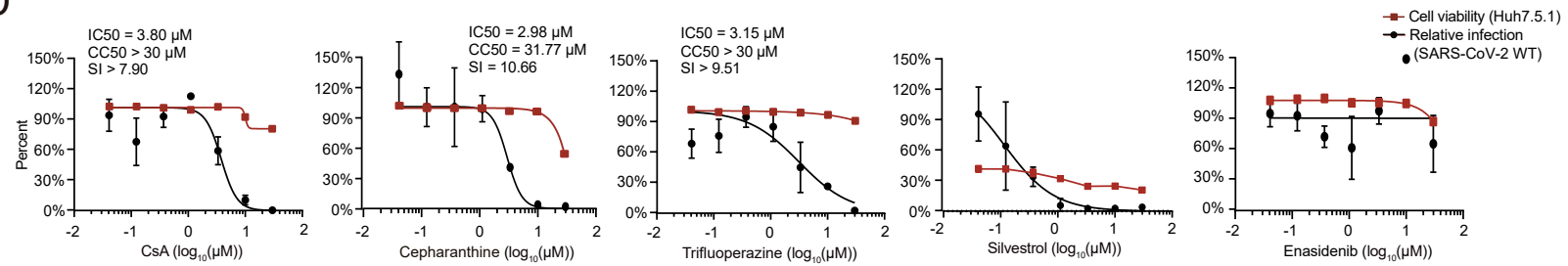

E
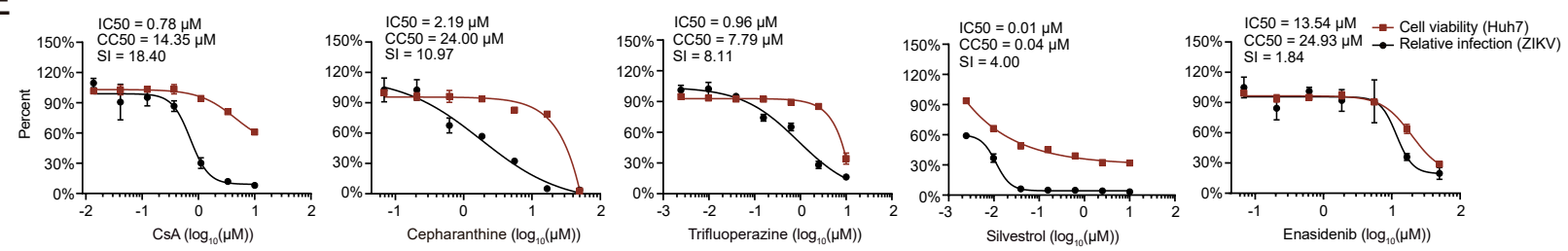

F
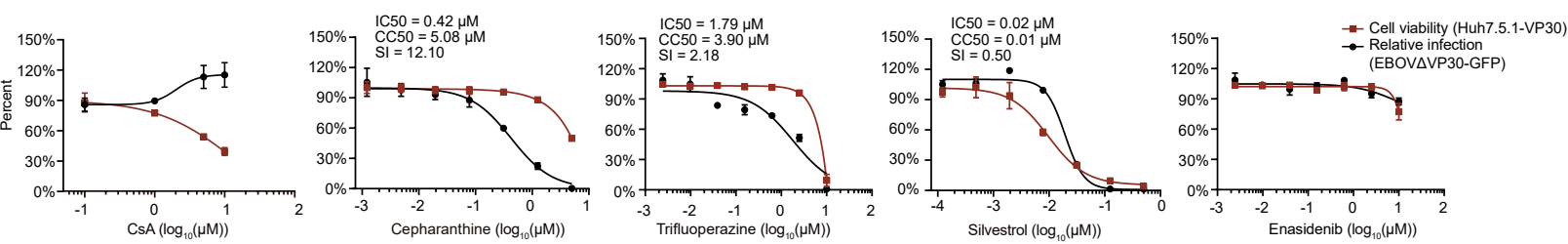
bioRxiv preprint doi: https://doi.org/10.1101/2021.04.25.441316; this version posted April 26, 2021. The copyright holder for this preprint (which was not certified by peer review) is the author/funder. All rights reserved. No reuse allowed without permission.

\section{Extended Data Fig. 1}

A

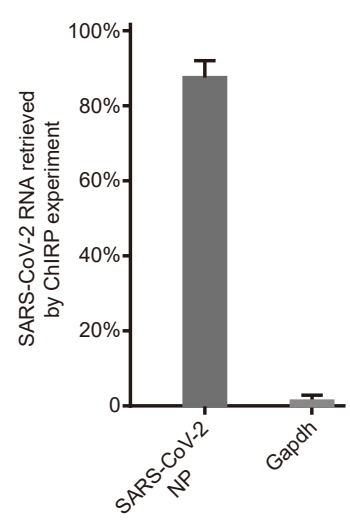

B

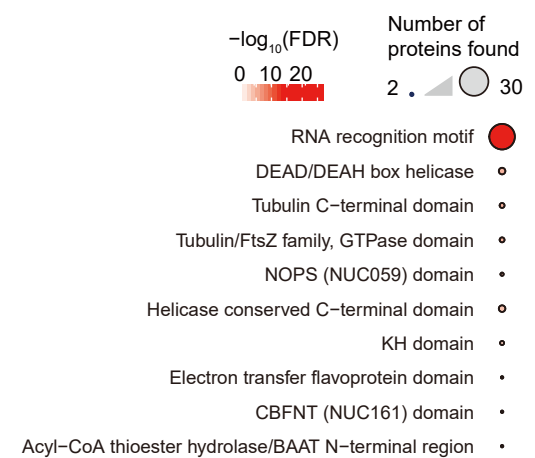

C

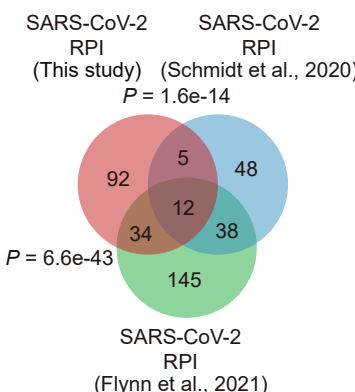

(Flynn et al., 2021) 
bioRxiv preprint doi: https://doi.org/10.1101/2021.04.25.441316; this version posted April 26, 2021. The copyright holder for this preprint (which was not certified by peer review) is the author/funder. All rights reserved. No reuse allowed without permission.

Extended Data Fig. 2

A

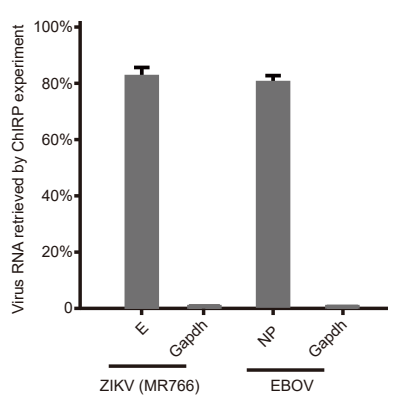

B

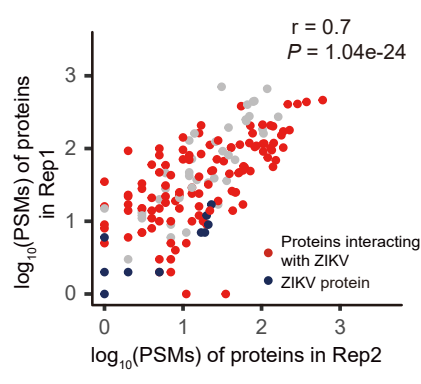

C

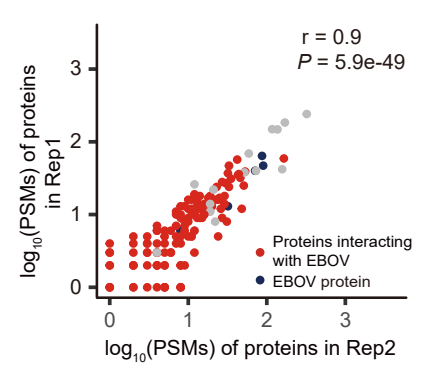

$\mathrm{D}$

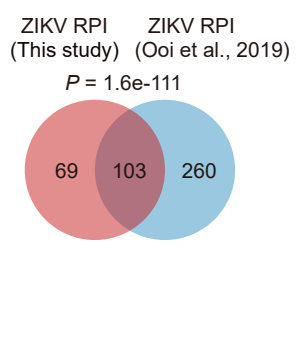


bioRxiv preprint doi: https://doi.org/10.1101/2021.04.25.441316; this version posted April 26, 2021. The copyright holder for this preprint (which was not certified by peer review) is the author/funder. All rights reserved. No reuse allowed without permission.

\section{Extended Data Fig. 3}

A

\begin{tabular}{|c|c|c|}
\hline \multirow{3}{*}{$\begin{array}{c}-\log _{10}(P \text { value }) \\
0123\end{array}$} & \multicolumn{2}{|c|}{$\begin{array}{l}\text { Number of } \\
\text { proteins found }\end{array}$} \\
\hline & $2 \circ \square$ & O 5 \\
\hline & TCA cycle & 0 \\
\hline \multicolumn{2}{|c|}{ Translational elongation } & 0 \\
\hline \multicolumn{2}{|c|}{ Microtubule-based process } & o \\
\hline \multicolumn{2}{|c|}{ mRNA splicing, via spliceosome } & 0 \\
\hline \multicolumn{2}{|c|}{ Gluconeogenesis } & 0 \\
\hline \multicolumn{2}{|c|}{ L-proline biosynthetic process } & $\circ$ \\
\hline \multicolumn{2}{|c|}{ Isocitrate metabolic process } & $\circ$ \\
\hline \multicolumn{2}{|c|}{ Proline biosynthetic process } & $\circ$ \\
\hline \multicolumn{2}{|c|}{ Citrate metabolic process } & $\circ$ \\
\hline \multicolumn{2}{|c|}{ Carbohydrate metabolic process } & 0 \\
\hline
\end{tabular}

D

$\begin{array}{rr}\text { ZIKV specific interacting proteins } \\ -\log _{10}(P \text { value }) & \begin{array}{c}\text { Number of } \\ \text { proteins found }\end{array} \\ 0369 & 5 \circ\end{array}$

B $\begin{array}{cc}\text { SARS-CoV-2 specific interacting proteins } \\ -\log _{10}(P \text { value }) & \begin{array}{c}\text { Number of } \\ \text { proteins found }\end{array} \\ \begin{array}{llll}2 & 4 & 6 & 3 .\end{array} 17\end{array}$

Citrate cycle (TCA cycle) • Biosynthesis of antibiotics 0 Biosynthesis of amino acids 。 Metabolic pathways Carbon metabolism 。

Glyoxylate and dicarboxylate metabolism 。 Pyruvate metabolism 。 Arginine and proline metabolism 。 Pathogenic Escherichia coli infection 。 2-Oxocarboxylic acid metabolism 。

E EBOV specific interacting proteins $-\log _{10}(P$ value $) \quad$ Number of $\operatorname{g}_{10}(P$ value $) \quad$ proteins found
01234 3 . $\bigcirc 10$

rRNA processing Translational initiation SRP-dependent cotranslational membrane targeting Retina homeostasis Nonsense-mediated mRNA decay Osteoblast differentiation 0 Translation $\mathrm{O}$

Keratinocyte differentiation 0 Sterol biosynthetic process 。
C

$$
\begin{array}{ccc}
\text { ZIKV specific interacting proteins } \\
-\log _{10}(P \text { value }) & \begin{array}{c}
\text { Number of } \\
\text { proteins found }
\end{array} \\
\begin{array}{llll}
0 & 8 & 16 & 4
\end{array}
\end{array}
$$

Translational initiation

Nonsense-mediated mRNA decay SRP-dependent cotranslational membrane targeting
mementationt Viral transcription rRNA processing Translation $\bigcirc$ Cell-cell adhesion

Negative regulation of translation $\circ$ Negative regulation of apoptotic process $\mathrm{O}$

$$
\text { Gene expression 。 }
$$

F

EBOV specific interacting proteins

$$
\begin{array}{cc}
-\log _{10}(P \text { value }) & \begin{array}{l}
\text { Number of } \\
\text { proteins found }
\end{array} \\
012 & 3 \circ \\
0
\end{array}
$$

Ribosome

Biosynthesis of antibiotics 。

Protein processing in ER $\circ$

Glycolysis/Gluconeogenesis 。

Systemic lupus erythematosus 。 Metabolic pathways 